\title{
COVID-19 Pneumonia: A Review of Typical Ct Findings and Differential Diagnosis.
}

\author{
Klaus Irion MD, Ph.D ${ }^{1}$, Ali Nawaz Khan, MBBS, MRCS, FRCR, FRCP1, Anthony Kodzo-Grey Venyo. MB, ChB, FRCS 2* \\ ${ }^{1}$ Consultant Radiologist, Prof. Hon. North Manchester General Hospital, Manchester M8 6RB. \\ ${ }^{2}$ Educational Supervisor Accreditation Certificate (RCP London). Urologist North Manchester General Hospital. M8 5RB. United Kingdom.
}

*Corresponding Author: Anthony Kodzo-Grey Venyo FRCS (Ed), FRCSI. North Manchester General Hospital, Department of Urology, Manchester M8, MRB. United Kingdom.

Received date: August 24, 2020; Accepted date: October 05, 2020; Published date: October 15, 2020

Citation: I Klaus, A N Khan, K-G V Anthony. (2020) COVID-19 Pneumonia: A Review of Typical Ct Findings and Differential Diagnosis. Biomedical Research and Clinical Reviews. 1(3); DOI: 10.31579/2692-9406/019

Copyright:@2020 Anthony Kodzo-Grey Venyo, This is an open-access article distributed under the terms of the Creative Commons Attribution License, which permits unrestricted use, distribution, and reproduction in any medium, provided the original author and source are credited.

\begin{abstract}
Pneumonia that is caused by the 2019 novel coronavirus (SARS-CoV-2, which is also referred to as 2019-nCoV recently did break out in Wuhan China has been coined the terminology of COVID-19. With the spread of the disease, similar cases of COVID-19 had been confirmed in various regions of the world. Because COVID-19 is a relatively new global disease, clinicians, and patients across the globe would initially not be conversant with the clinical features and radiology imaging characteristics of SARS-CoV-2 pneumonia. The causes of pneumonia are protein, many secondary to an underlying cardiorespiratory abnormality while some are related systemic disease. Various imaging techniques generally diagnose cases of Pneumonia. In the current climate, COVID-19 Pneumonia has taken center stage; confirmation relies upon microbiological studies such as realtime polymerase chain reaction or sequencing. These investigations are not usually available in an emergency setting. Computed Tomography (CT) can be used as an essential complement for the diagnosis of COVID-19 Pneumonia in the current epidemic context. But the later may be misleading as other cases of Pneumonia, and interstitial lung disease can easily be confused with COVID-19 Pneumonia. Also, Covid19 Pneumonia may be missed if not considered. The attention of clinicians should be alerted to the possibility of COVID-19 to conduct the appropriate tests to confirm or negate the diagnosis of COVID-19. In asymptomatic as well as in symptomatic patients that have COVID1-9 pneumonia, the initial COVID-19 nuclei acid test results could be normal, which upon subsequent repeat testing would become normal. Still, radiology imaging using a CT scan of thorax would tend to demonstrate various non-specific features that affect a variable number of lobes of the lungs, and these features quickly increase in size when a repeat CT scan of the thorax is undertaken. These findings tend to predate positive COVID-19 test results in some cases of COVID-19. The non-specific changes tend to resolve when the patient resolves from COVID-19 pneumonia. A catalog of radiology images that demonstrate various types of cardio-pulmonary lesions which when encountered by clinicians should alert them to exclude the possibility of COVID-19 Pneumonia has been included in the paper as an aid to alerting clinicians to have a high index of suspicion of radiology images of the thorax which should help them to quickly undertake appropriate tests to confirm or negate the diagnosis of COVID-19 pulmonary infection...
\end{abstract}

Key Words: pneumonia; differential diagnosis; imaging; covid-19 pneumonia

\section{Introduction}

Pneumonia is a common illness and no respecter for age or gender. Imaging is generally the prime investigation. In the present, climate consideration of Covid19 is essential, and early diagnosis can be lifesaving. Corona Virus pandemic is an extremely new medical condition that has pandemically affected the entire world suddenly with recordings of varying incidence rates and deaths in all the continents and countries in the world; it would be envisaged that both clinicians of all specialties including radiologists and patients would tend not to be familiar with the clinical manifestations, laboratory investigation diagnostic features of the disease. It is also likely that some radiologists globally would not have encountered cases of Covid19 pneumonia/pneumonitis before; therefore, it would be possible that these radiologists would tend not to be familiar with the radiology imaging features of the disease.

It has been emphasized that the standard of reference for confirming COVID-19 relies on microbiological tests such as real-time polymerase chain reaction (RT-PCR) or sequencing. However, these tests might not be available in an emergency setting. Computed tomography (CT) can be used as an essential complement for the diagnosis of COVID-19 pneumonia in the current epidemic context. In this review, we present the typical CT features of COVID-19 pneumonia and discuss the main differential diagnosis. [1] The cases of COVID19 Pneumonia reported 
worldwide have been pitched at 20.9M, of which $13 \mathrm{M}$ have recovered, and $760 \mathrm{~K}$ lost their life. [2]

\section{Aim}

To review the selected data of COVID19 Pneumonia with a particular emphasis on imaging and differential diagnosis of COVID19 Pneumonia and its mimics.

\section{Method}

PUBMED, Yahoo, Google, Google Scholar, and other search engines were searched were used, and references were utilized to write the review of the literature. Additionally, we used our data from Klaus et al. to provide various cardiopulmonary radiology images when encountered by clinicians to alert them to undertake appropriate tests to confirm or negate the diagnosis of Covid19 and to ensure a correct diagnosis is quickly established.

\section{Results / Literature Review}

(A) Miscellaneous narrations and discussions from some case report case series, and studies related to Covid19 pneumonitis/pneumonia.

Mimics and chameleons of COVID-19 Pneumonia: [3].

Nickel and Bingisser [3] emphasized that mimics are pathological/physiological entities that closely simulate a remote object, an example being unilateral weakness due to hypoglycemia that mimics a stroke. Nickel and Bingisser [3] also stated that, on the other hand, chameleons often tend to be overlooked or confused with or mistaken for existing underlying pathologies. Nickel and Bingisser [3] additionally stated that within the present-day context, it is essential/vital that "mimics and chameleons" are understood so as not to confuse them with COVID19 Pneumonia.

Nickel and Bingisser [3] reported an 83-year-old patient who was transferred to the emergency department according to having sustained a fall. As he had sustained thoracic trauma, a CT scan of his chest was performed, which revealed bilateral, sub-pleural, ground-glass opacities with air bronchograms. The patient was afebrile and did not have a cough. Fortunately, he was immediately diagnosed with COVID-19 and admitted. Nickel and Bingisser [3] emphasized that the first case of Covid-19, in Basel, Switzerland, was first reported on the $25^{\text {th }}$ of February 2020, which was just a few days pursuant to the first report of Covid-19 in Italy. Based upon the fact that Covid-19 had previously not been encountered, their experience with Covid-19 was short and that lessons need to be learned quickly from experience gathered concerning the treatment of such cases. Nickel and Bingsser [3] pointed out that a recent study had illustrated that abnormal lung computed tomography (CT) scan findings could be present in "asymptomatic" patients who are suffering from COVID-19 pneumonia [4] Nickel and Bingisser [3] pointed out that such results were recorded by Xie et al. [5] even before viral RNA became detectable Nickel and Bingisser [3] explained that the recent recognition could explain the finding of CT scan findings of abnormalities within the lung preceding the viral RNA detection that current tests do lack sensitivity. Hence, infections could, therefore, be missed [6] Nickel and Bingisser [3] made the ensuing summations related to COVID-19 infections:

- In addition to the problem concerning the sensitivity test for the establishment of the diagnosis of COID-19, they had been facing the issue of the sensitivity of the clinical features of the disease, which does represent the cornerstone of all considerations relating to testing of patients and the isolation of patients.
- Currently, they were treating three patients who had been suffering from severe COVID-19 and who were asymptomatic at their initial manifestation and hence some authors had emphasized that there is the need of a new framework and new definitions for this group of asymptomatic patients who are older and who tend to manifest with non-specific symptoms such as weakness and fatigue [3] [7]

- It has been emphasized that these clinical manifestations could be coined or referred to as "COVID-19 chameleons" in analogy to the concept of "stroke chameleons," which should encompass expressions that do not appear to represent stroke initially, do subsequently emanate as an acute stroke. [3] [8]

- It was their opinion that the differential diagnosis in with "everything and nothing" must include "COVID-19 chameleon" as based upon their observations, which should not be regarded as an isolated case. It needs to be noted that "COVID-19 chameleons" do not appear to be confined to frail older patients only, and it should be realized the disease could also affect younger individuals.

Nickel and Bingisser [3] reported another patient who was an 80-year-old woman who had been living in a residential care home who had manifested in the emergency department with dyspnoea as well as cough. The referring family physician suspected that the patient might have COVID-19 because two other residents within her care home had already been diagnosed as having COVID-19 infection. According to extensive assessment workup, she was diagnosed as having decompensated heart failure as the most likely cause of her diffuse lung infiltrates because she had nasal as well as pharyngeal swabs, and their study demonstrated negative results for SARS CoV2. Nickel and Bingisser [3] believed that the second case could be named or referred to as "COVID-19 mimic" in analogy to "stroke mimics," which are presentations of non-vascular disease manifesting with a stroke-like clinical picture as coined by Huff. [8] Other summations made by Nickel and Bingisser [3] included:

- Because the numbers of coronavirus infections have been rising exponentially, there is an urgent global need to develop a test management protocol guidelines for older patients. They have "specific" as well as non-specific" complaints to provide the optimal or best care of patients, efficient use of resources as well as the protection of proxies.

- It has been documented that some healthcare systems had experienced exponential growth concerning COVID-19 cases; nevertheless, other healthcare systems had been able to retain a linear growth pattern.

- The recognition of cases with the utilization of clinical skills as well as early isolation of patients does represent one of the cornerstones of concerning the fight against the pandemic; nevertheless, testing is another way of minimizing the number of cases of COVID-19 infections, and this has been emphasized by [9]

- It has been emphasized that the present policy of various government bodies to focus upon patients who are at risk for the development of COVID-19 who have specific symptoms has also been limited by the concept of "chameleons," such as patients who have non-specific symptoms. It has been suggested that since the denominator for the development of COVID-19 is not known, the extension of testing patients who present with possible COVID-19 mimics should be taken into consideration. [3] [10]

Based upon lessons learned from the summations of Nickel and Bingisser [3] it would be argued that there an urgent need to investigate asymptomatic individuals who are asymptomatic and have undergone radiology imaging that has shown abnormalities within the lungs as well 
as individuals who have cardiopulmonary symptoms who have been revealed based upon radiology imaging to have various abnormalities within their lungs, pleura, and pericardium to ascertain quickly if they have COVID-19 Pneumonia so that they can be supported medically as well as isolated early to avoid other individuals getting COVID-19 infection. The lesson learned from the summations also illustrates that clinicians need to be aware that several patients who are shown to have cardiopulmonary lesions may not have COVID-19 infection at all and early testing to exclude COVID-19 in this COVID-19 pandemic era would re-assure individuals who do not have COVID-19 but rather cardio-pulmonary disease.

Li et al. [11] stated that the outbreak of corona virus disease (COVID-19) has made the medical management of colorectal cancers difficult. Li et al. [11] made summating iterations related to cancer patients and coronavirus as follows:

- Patients who have cancer would tend to be more susceptible to infection Cancer, as well as a history of the tumor, has been defined as an essential factor that relates to poor prognosis, which would tend to be challenging to both doctors and patients.

- Concerning patients who have metastatic colorectal carcinoma (MRC), maintenance treatment is optimal for choice management of such patients.

- Concerning patients who have a progression of tumor or poor biological behavior of tumors, they should receive combination chemotherapy or if they have been continued on combination chemotherapy.

- The utilization of adjuvant chemotherapy should reduce the intensity of the treatment of the patients, and it should shorten the time of treatment.

- Individual patients who are found to be febrile during their chemotherapy would be assessed concerning the possibility of differential diagnosis as well as they should be screened based upon the national standards of each country.

- About patients who are found to have stable diseases and who are in good general conditions associated with good performance status, their radiology imaging should be delayed until a later date.

- Clinicians should make individual clinical decisions based on the specifics of each patient during the COVID-19 pandemic situation. Lessons learned from the summations of Li et al. [11] include the fact that clinicians, surgeons, anesthetists, and radiologists have a pivotal role to play about adjustments that must be made to the timing of radiology imaging, and various treatment options of their patients to minimize and avoid the spread of COVID-19 pandemic but also selectively undertaking emergency and life-saving procedures for individual patients who would die if they are not provided emergency / urgent care. Some individuals who have cancer and may require immediate attention but are asymptomatic may have respiratory tract infection, or they could be asymptomatic but have COVID-19, and they would need selective, careful radiology imaging under precautions. Some of these steps include the following:

- Patients who are referred to the urology team because of nonvisible hematuria would have their cases discussed by the multidisciplinary team of urologists, radiologists, and pathologists who would state that majority of individuals who have nonvisible hematuria following investigation would be found not to have any abnormality. They would be discharged, and the few individuals who have non-visible blood in their urine who have lesions in their urinary tract would tend to have tiny lesions that would not kill them within the next 6 months to 12 months so their radiology imaging of $\mathrm{CT}$ urography and flexible cystoscopy would be postponed for 6 months, and this would be communicated with the patients in a non-contact way by telephone/letter to avoid unnecessary contact and invasive procedure during the COVID-19 pandemic to prevent.

- Patients who have visible hematuria are made to undergo a noncontrast CT scan of the abdomen and pelvis / renal tract, and this procedure would always be done keeping the necessary preventive distance, which does not allow contact between the patient and the radiographer. If there is no evidence of a large tumor in the renal tract seen on the images, the patient will not undergo flexible cystoscopy or any invasive urology procedure for the next 6 months during the COVID-19 pandemic. Nevertheless, if there is a large lesion that could be muscleinvasive that could be ensued by death of the patient or metastases within the next few months then the multidisciplinary team would request a non-contrast CT Thorax and also before a decision is taken to undertake any life-saving procedure a COVID-19 test would be conducted. The individual who does not have any lung lesion as well as who has a negative COVID-19 test result would be carefully scheduled to undergo treatment for the disease. Individuals who are found to be asymptomatic but have radiology imaging of any significant lung lesion would also be screened for COVID19, and those who have COVID-19 would be referred appropriately to the infectious disease COVID-19 team and would need to be quickly isolated. Those who have negative COVID-19 test results but have pulmonary lesions would be referred to the chest physician for further assessment. This exercise does illustrate the role of the radiology team in the noncontact evaluation of patients in the COVID-19 pandemic era.

- Some individuals tend to be referred by their General Practitioners to the Urology Team based upon ultrasound scan finding of a lesion within the kidney, urinary bladder, or prostate for assessment. The multi-disciplinary team of radiologists, urologists, and pathologists would review the images and if the lesions are not suspicious they tend to decide to re-assure the patients in a non-contact way by telephone/letter re-assuring them they have Bosniak type 2 cyst or a benign lesion which does not need any further investigation for re-assurance. If the ultrasound scan was undertaken in the district and the images are not available in the radiology PACS system then non-contrast CT scan of the abdomen and pelvis / renal tract is requested which would be undertaken carefully without contact between the radiographer/radiologist and the patient and if upon reviewing the images, there are no suspicious lesions the patients would be discharged pursuant to non-contact telephone discussion plus by a letter. Some lesions may look suspicious, but if they are small and would generally not be ensued by any quick progress or harmful effects, then non-contract further imaging could be requested to be undertaken after variable intervals which could vary from 6 weeks to 3 months and sometimes 6 months. Individuals who have lesions that are large or significant that could be malignant or portend a quick progress and possible death that would require an urgent surgical operation to avoid death, then noncontrast CT scan of thorax would be requested and undertaken in a non-contact way as well as COVID-19 test would be conducted to exclude COVID-19. CT Thorax could demonstrate a pulmonary lesion that is not related to COVID19 , or it could reveal cardiopulmonary lesions that could be associated with COVID-19 that should be carefully investigated. Acutely ill patients who are found to have COVID-19 that require urgent surgical treatment would need to 
recover well before they can undergo surgery. The multidisciplinary team would always discuss knowledge of various cardiopulmonary diseases that are picked up by the radiologist with the discussion led by the radiologist, and an appropriate referral would be made to the physician, and the surgery would be postponed.

These decisions by urologists and other surgeons do always rely upon radiology imaging findings of radiologists who always discuss their results collaboratively with the multi-disciplinary team.

Chen et al. [12] analyzed the clinical characteristics of 2019 novel coronavirus (2019-nCoV) pneumonia as well as to ascertain the correlation between the results of serum inflammatory cytokines and the severity of the disease. Chen et al. [12] emphasized that 29 patients who had 2019-ncov had been admitted to the isolation ward of Tongji Hospital that was affiliated to Tongji Medical College of Huazhong University of Science and Technology in January 2020 who had been selected as subjects of the study. Chen et al. [12] collected the clinical data, and they analyzed the general information, clinical symptoms, the results of the blood tests, as well as the computed tomography (CT) scan imaging features of the patients. Based upon relevant diagnostic criteria, the patients were subdivided into three groups as follows: (a) mild disease group that included 15 patients, (b) severe disease group that consisted of 9 patients, and (c) critical disease group that comprised of 5 patients. They did analyze the expression levels of inflammatory cytokines, as well as other markers within the serum of each group of patients, were detected, and the changes of these indicators of the three sub-groups were compared, including their relationship with the clinical sub-classification of the disease. Chen et al. [12] summated the results as follows:

- (1) The main manifesting symptoms of 2019-nCoV pneumonia were fever, which was reported in 28 out of the 29 patients, which was associated with or without respiratory tract symptoms. Two of the patients died as a sequel to the underlying disease and co-bacterial infection, respectively.

- (2) The results of the blood tests of the patients did demonstrate normal or decreased white blood cell count in 23 out of the 29 cases, decrease in the lymphocyte count in 20 out of the 29 cases, increased hypersensitive $\mathrm{C}$ reactive protein (hs-CRP) in 27 out of the 29 patients, as well as normal levels of procalcitonin. About the majority of the patients, the level of lactate dehydrogenase (LDH) was noted to be significantly increased, which had been observed in 20 out of the 29 cases, the level of serum albumin was decreased in 15 patients out of the 29 patients. Nevertheless, no significant changes were found in the results of Alanine aminotransferase (ALT), aspartate aminotransferase (AST), total bilirubin (Tbil), serum creatinine (Scr) as well as in the results of other items.

- (3) Computed tomography (CT) scan findings of typical 2019$\mathrm{nCoV}$ pneumonia were found to include single or multiple patchy ground glass shadows that are associated with a septal thickening. It was also found that as $2019-n \mathrm{CoV}$ pneumonia progressed, the lesion did increase in size, and the scope of the lesion did expand, and the ground glass opacity shadow was noted to co-exist with a solid shadow or a striped shadow.

- (4) Statistically significant differences were found about the expression of the levels of interleukin-2 receptor (IL-2R) and IL-6 within the serum of the three groups of patients with a Pvalue of $\mathrm{P}<0.05$, among which the critical disease group was higher in comparison with the severe disease group and the severe disease group was higher in comparison with the mild disease group of patients. Nevertheless, no statistically significant differences were found about the serum levels of tumor necrosis factor-alpha (TNF- $\alpha$ ), IL-1, IL-8. IL-10, hsCRP, lymphocyte count as well as LDH among the three disease groups of mild disease, severe disease, and critical disease with a P-value of $\mathrm{P}>0.05$. Chen et al. [12] made the ensuing concluding iterations:

- The clinical features of 2019-nCoV pneumonia tend to simulate those of common viral pneumonia.

- The undertaking of high-resolution computed tomography (CT) scan is of great value about the differential diagnosis of 2019nCoV pneumonia.

- The increased expression of IL-2R, and IL-6 within serum would be expected to predict the severity of the of 2019-nCoV pneumonia as well as the prognosis of individuals that are affected by 2019-nCoV pneumonia

Based upon lessons learned from the case publication of Chen et al. [12] it would be argued that utilization of high-resolution CT scan of the thorax in asymptomatic individuals and individuals who manifest with respiratory tract symptoms who are affected by $2019-\mathrm{nCoV}$ pneumonia would tend to demonstrate various lesions within the lung that are not specific to 2019-nCoV pneumonia including ground-glass opacities associated with other lesions but which should alert the clinician to be mindful of instituting tests that would confirm or negate the diagnosis of 2019-nCoV pneumonia. Within the developed countries, computed tomography (CT) scanners and magnetic resonance imaging (MRI) scanners tend to be readily available. Hence, various small cardiopulmonary lesions could be easily identified by radiologists and clinicians. Nevertheless, within some parts of developing countries, CT scans and MRI scans are not available. If they are available, they tend to break down, and the repair work on as well as restoration of the scanners to full functioning states tend to be delayed. For this reasons, it would be envisaged that perhaps some radiologists and clinicians may not be conversant with the features of ground-glass opacities and other subtle, as well as overt lesions which though not diagnostic of 2019-nCoV pneumonia, should alert all clinicians to undertake investigations that would confirm or negate the diagnosis of 2019-nCoV pneumonia to ensure those who have 2019-nCoV pneumonia are isolated and treated. Those who do not have 2019-nCoV pneumonia are re-assured. Within the latter part of this article, we have included various radiology imaging features which though not specific to $2019-\mathrm{nCoV}$ pneumonia should alert clinicians to the fact that they should exclude the possibility of 2019$\mathrm{nCoV}$ pneumonia whether the individuals are asymptomatic, or they have respiratory tract symptoms whether or not they also have a malignant disease or any other disease elsewhere.

An et al. [13] had stated that from December 2019 to the time of the report of their cases, the outbreak of the novel coronavirus had impacted nearly more than 90,000 individuals in more than 75 countries. An et al. [13] had aimed to define within their case report the chest computed tomography (CT) scan findings of 2019-novel coronavirus that was associated with pneumonia as well as its successful resolution under treatment. An et al. [13] reported a fifty-year-old woman who is a businesswoman who had manifested with the main symptom of fever over the preceding one week, diarrhea, anorexia, as well as asthenia". In the first instance, she was given Tamiflu treatment. She had influenza A virus serology, and the result was negative. She was commenced on levofloxacin 3 days subsequently because her symptoms had not improved. She had the novel coronavirus nucleic acid test, which was negative. It was noted that before the onset of her disease, she had been to Wuhan on a business trip. Despite having taken her levofloxacin medicament, her symptoms had not improved in that she was continued to have pyrexia, and her body temperature had risen to $39.2^{\circ} \mathrm{C}$. Given this, she was referred to the clinic of the authors for further assessments. She had a computed tomography (CT) scan of the thorax, which demonstrated bilateral multi-focal ground-glass opacities that were associated with consolidation, which was suggestive of viral pneumonia as a differential diagnosis based upon the radiology imaging features of her pulmonary lesions. She had a subsequent test that demonstrated that her 2019-novel coronavirus pneumonia nuclei acid test result was positive, which established a definite diagnosis of 2019-novel 
coronavirus pneumonia. Her 2019-novel coronavirus pneumonia was resolved under her treatment. An et al. [13] concluded that computed tomography (CT) scan of thorax does offer fast and convenient evaluation of patients who are suspected of having 2019-novel coronavirus pneumonia. Some of the lessons learned from this case report include the following:

- 2019-novel coronavirus tends to manifest with non-specific symptoms including (a) fever, (b) diarrhea, (c) anorexia, (d) and asthenia and based upon this all clinicians as well as patients should be aware that the aforementioned non-specific symptoms, as well as symptoms of difficulty in breathing and other respiratory tract symptoms, could be possibly related to the 2019-novel coronavirus pneumonia and they should have a high suspicion for the disease in this era of the 2019-novel coronavirus pneumonia pandemic to ensure prompt investigations are undertaken to confirm or negate a diagnosis of the disease.

- A sensitive and complete history should be obtained from all patients who present with non-specific symptoms including their travel history and whether they had been recently in contact with any 2019-novel coronavirus individual or a place where the incidence of the disease has been high to ensure all patients are promptly investigated for the disease.

- It needs to be noted that in the initial phase of the disease, the results of the 2019-novel coronavirus pneumonia nuclei acid test would tend to be normal. Subsequently, the results would become normal. Therefore, an initial negative effect does not exclude the disease, and given this, a second test needs to be undertaken subsequently to confirm or negate the diagnosis of 2019-novel coronavirus pneumonia.

- Initially when the results of the 2019-novel coronavirus pneumonia nuclei acid test are normal in the early phases of the disease, computed tomography (CT) scan of thorax would tend to demonstrate evidence of ground-glass opacities and other lesions which should alert the radiologists and clinicians to the possibility of 2019-novel coronavirus pneumonia so that tests are undertaken to confirm or negate the diagnosis as well as with a high index based upon non-specific radiology imaging findings precautionary isolation of patients should be initiated. The role of the radiologist in picking up non-specific radiology image features that could represent the disease in its infancy has been pointed out.

Zhang et al. [14] reported a 64-year-old man who had presented with a recurrent fever after he had caught a cold for 6 days. He stated that he had felt better undertaking anti-febrile agents. One day preceding his visit to the hospital, he developed fever again as well as dizziness, headache, aching of his four limbs, but no cough, shivering, tightness of chest, chest pain, nausea, and vomiting, et cetera. About travel history, he stated that he had traveled from Wuhan to Kunming. He did not have any significant past medical accounts, and he had never had hepatitis, tuberculosis, typhoid before as well as he had not been in contact with poultry or mosquito bites. He was admitted to the hospital of the authors on the $21^{\text {st }}$ of January 2020, and he still had fever following his admission, with his highest temperature recorded as $39.2^{\circ} \mathrm{C}$. He had a real-time RT-PCR test, which revealed positive results for the 2019 novel coronavirus (2019$\mathrm{nCoV})$. The undertaking of microbiology tests excluded the possibility of the man having combined infection with other pathogens, and the results were normal. He had a CT scan of thorax, which demonstrated features that simulated the CT scan of thorax features that had been previously described by Huang et al., [15] and also by Pan et al. [16] (see figure for the CT thorax of the patient). The CT scan of the thorax showed multiple patchy ground-glass opacities (GGOs in both lungs and the majority of the opacities were in sub-pleural areas. Follow-up CT scans of the thorax demonstrated that within 3 days, nodular GGOs were also revealed together with sub-pleural patchy GGOs. Multiple patchy ground-glass opacities (GGOs) were seen in bilateral lung, mostly in subpleural areas. They progressed within 3 days, and nodular GGOs were also seen together with subpleural patchy GGOs. Zhang et al. [14] emphasized that knowledge of the computed tomography (CT) scan features of COVID19 pneumonia could be helpful about the early diagnosis and treatment of patients as well as in the control of the spread of the infection which in this case would involve isolation of the patient. Zhang et al. [14] made the ensuing conclusions:

- Their case of COVID-19 pneumonia had demonstrated multiple subpleural ground-glass opacities (GGOs) in both lungs, rapid progression, and it also did accompany nodular GGOs upon the CT scan of the thorax.

- Their findings were consistent with the results that had been previously reported, as well as they might be useful about early detection as well as for the evaluation of the severity of COVID-19 pneumonia.

Lessons learned from this case report include the following:

- The presentation of Covid-19 pneumonia tends to be nonspecific, and these manifestations mimic other conditions that are more commonly encountered. Therefore, a high index of suspicion of COVID-19 pneumonia is pertinent to the early diagnosis of the disease.

- $\quad$ Some of the CT thorax findings that tend to be visualized early include: (a) ground-glass opacities within the one lung or both lungs, (b) ground-glass opacities within the sub-pleural regions.

- Repeat CT scans of the thorax a few days following an initial CT of the thorax of some patients that have COVID-19 pneumonia may demonstrate an increase in size as well as numbers of the ground glass opacities.

- The finding of ground-glass opacities within the lung or lungs, as well as within the sub-pleural regions of asymptomatic as well as symptomatic individuals, should alert clinicians quickly to the possibility of COVID-19 pneumonia so that they can undertake tests that would confirm or negate the diagnosis of COVID-19 pneumonia. In fact, in this COVID-19 pandemic, everyone who is found to have ground-glass opacity within the lung or sub-pleural region should undergo the COVID-19 test to ascertain if the individual has COVID-19 pneumonia or not quickly.

It is well known that generally, all hospital establishments within the developed countries have facilities for the undertaking of computed tomography (CT) scans of the thorax as well as magnetic resonance imaging (MRI) scans of the thorax and therefore ground glass opacities would be picked up quickly within the hospital establishments. It is also well known that within many hospitals in the developing countries where COVID-19 pneumonia cases are also being reported, CT scan and MRI scan facilities are usually not available and in the few hospitals where CT scan and MRI scan facilities are available, quite often the CT scan machines, and the MRI scanners tend to break down often. The repair work, as well as the normal return to function of the scanners, tend to be delayed, and only chest $\mathrm{x}$-rays tend to be most commonly available for the assessment of respiratory tract symptoms initially. It is also known that chest $\mathrm{x}$-rays generally are not capable of detecting small ground-glass opacities which does mean that if a patient has COVID-19 pneumonia and presents to the hospital, there would tend not to be facilities for CT scan or MRI scan which would expect that early detection of ground-glass opacity in such hospitals would be impossible. Hence, CT scan and MRI scan cannot be used as a radiology imaging means of detecting possible pulmonary lesions that could represent COVID-19 pneumonia and for this reason, clinicians need to be advised that if patients present with nonspecific symptoms, they should have a high index of suspicion for COVID-19 pneumonia in the absence of any significant chest $\mathrm{x}$-ray findings and they should quickly undertake COVID-19 tests for all their 
patients. In such scenarios, even if the result of the first test is negative, the patients should be initially treated but isolated, and a repeat COVID19 test should be subsequently undertaken to confirm or negate the diagnosis of COVID-19. Considering the usefulness of CT scans in the early detection of ground-glass opacities, some of which could be related to COVID-19 and some of the ground-glasses could subsequently enlarge, it would be argued that during this COVID-19 pandemic, Governments in developing countries should find a way of getting more CT scans for hospitals that do not have CT scans as well as communities that do not have CT scans should make efforts to undertake self-help money-raising efforts to obtain sufficient money to acquire CT scans for the benefit of their health as well as extra money for the maintenance of the CT scans as a collaborative effort to support the efforts of their Governments and district councils.

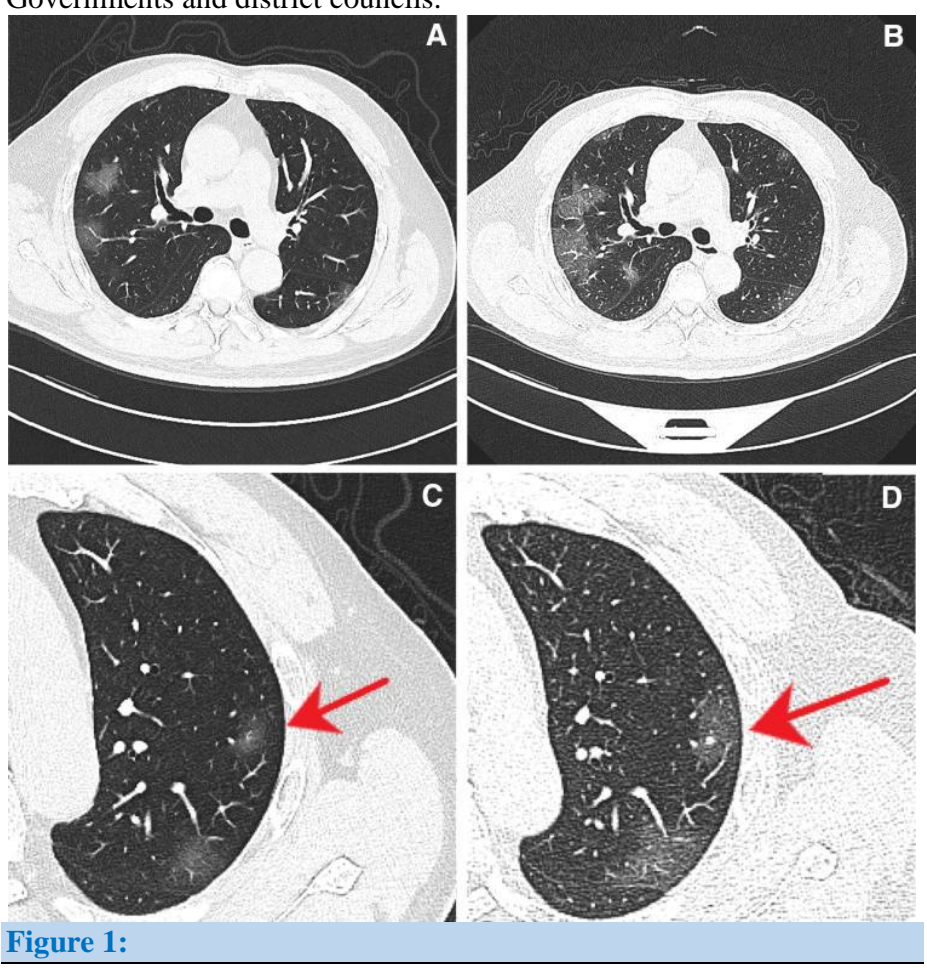

A: Chest CT on admission shows multiple ground-glass opacities in bilateral lungs, mainly in the subpleural areas. B: CT image of 3 days later shows a marked progression of multiple ground-glass opacities in subpleural areas. C, D: High-resolution CT images at the same period of $\mathrm{A}$ and $\mathrm{B}$, respectively. They show nodular ground-glass opacities in the subpleural areas in the upper lobe of the left lung, and they significantly progressed within 3 days. Reproduced from: [14] Zhang X, Song W, Liu X, Lyu L. CT image of novel coronavirus pneumonia: a case report. Jpn J Radiol. 2020 Mar 18: 1-2. DOI: 10.1007/s11604-020-00945-1. https://www.ncbi.nlm.nih.gov/pmc/articles/PMC7088615/ under copyright (c) Japan Radiological Society 2020 This article is made available via the PMC Open Access Subset for unrestricted research reuse and secondary analysis in any form or by any means with acknowledgment of the source. These permissions are granted for the duration of the World Health Organization (WHO) declaration of COVID-19 as a global pandemic.

Huang et al. [15] reported a 36-year-old man who had manifested with a 2-days history of fever, sore throat, as well as fatigue 5 days pursuant to his visiting Wuhan, in China. His temperature upon his admission was 37.8 degrees centigrade $\left(100.04^{\circ} \mathrm{F}\right)$. The examination of his respiratory system was normal, with normal sounds upon consultation. The results of his laboratory blood tests were normal in that his white blood cell count was $4.6 \times 10^{9} / \mathrm{L}$, which was normal, and his differential blood count revealed $53.1 \%$ neutrophils. His blood procalcitonin level was within a normal range. He had a computed tomography $(\mathrm{CT})$ scan of thorax, which revealed multiple peripheral ground-glass opacities within both of his lungs, as well as the CT scan, demonstrated more involvement of his left upper lobe as well as the lingular segment (see figures 2 a to c). During his admission, he had a real-time fluorescence polymerase chain reaction (RT-PCR) assay of his sputum, and the result was negative for the 2019 novel coronavirus (2019-nCoV) nuclei acid. He had a repeat computed tomography (CT) scan of thorax 3 days following his admission, which demonstrated the transformation of ground-glass opacities to more consolidation (see figures $2 \mathrm{~d}$ to $2 \mathrm{f}$ ). He also had a repeat RT-PCR 2019nCoV nucleic acid assay, and the result at that time was negative. Six days pursuant to his admission, he had a third RT-PCR 2019-nCoV nucleic acid assay, and the result was finally shown to be positive. Huang et al. [15] emphasized that when the specimen tests bring out negative results, the possibility of a false-negative result should be taken into consideration concerning the recent exposures of the patient as well as the presence of clinical signs and symptoms that are consistent with 2019-nCoV infection, [17] [18] [19] in addition to the history of recent exposure which did indicate that $2019-\mathrm{nCoV}$ infection was the likely diagnosis.

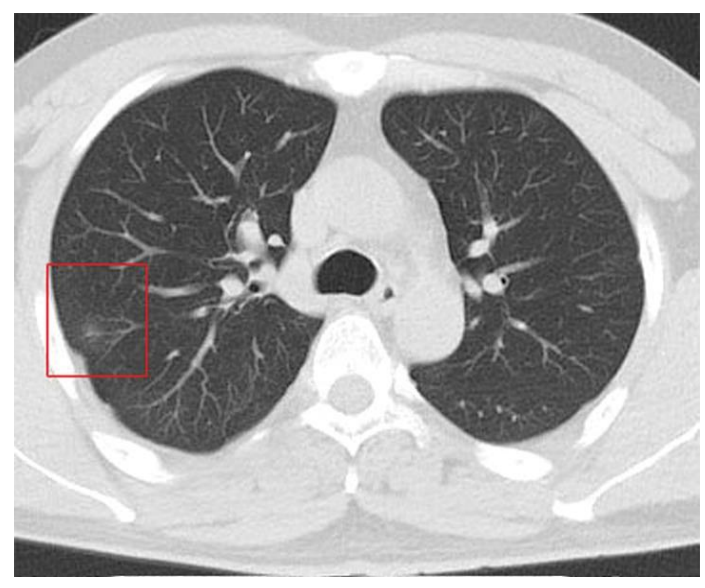

\section{Figure 2a:}

Figure 1: Images in a 36-year-old man with a 2-day history of fever, sore throat, and fatigue 5 days after visiting Wuhan, China, and a negative sputum real-time fluorescence polymerase chain reaction assay for the 2019 novel coronavirus. (a, b) Chest CT scans obtained at presentation show ground-glass opacities (red box) in the right upper lobe, and the lingular segment and the left lower lobe (b). (c) Volume rendering of a chest CT scan obtained at admission. (d, e) CT scanned obtained 3 days after admission show progression of ground-glass opacities to an atoll sign in the right upper lobe (red boxes in d) and left lower lobe consolidation (red boxes in e). (f) Volume rendering of the chest CT scan obtained 3 days after admission shows the new areas of consolidation. They are reproduced from [15] Huang P, Liu T, Huang L, Liu H, Lei M, $\mathrm{Xu} \mathrm{W}, \mathrm{Hu} \mathrm{X}$, Chen J, Liu B. Use of Chest CT in Combination with Negative RT-PCR Assay for the 2019 Novel Coronavirus but High Clinical Suspicion. Radiology. 2020;295(1):22-23. doi:10.1148/radiol.2020200330

https://pubmed.ncbi.nlm.nih.gov/32049600/ under Copyright 2020 by the Radiological Society of North America, Inc.

This article is made available via the PMC Open Access Subset for unrestricted re-use and analyses in any form or by any means with acknowledgment of the source. These permissions are granted for the duration of the COVID-19 pandemic or until permits are revoked in writing. Upon expiration of these permissions, PMC is granted a perpetual license to make this article available via PMC and Europe PMC, consistent with existing copyright protections. 


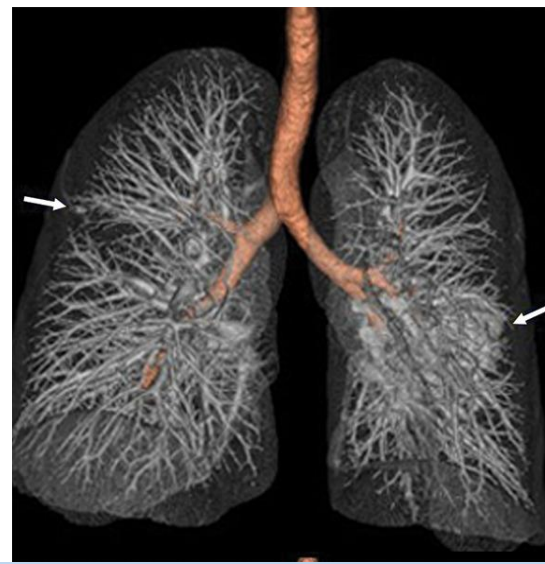

Figure 2b:

Figure 2b Images in a 36-year-old man with a 2-day history of fever, sore throat, and fatigue 5 days after visiting Wuhan, China, and a negative sputum real-time fluorescence polymerase chain reaction assay for the 2019 novel coronavirus. (a, b) Chest CT scans obtained at presentation show ground-glass opacities (red box) in the right upper lobe, and the lingular segment and the left lower lobe (b). (c) Volume rendering of a chest CT scan obtained at admission. (d, e) CT scanned obtained 3 days after admission show progression of ground-glass opacities to an atoll sign in the right upper lobe (red boxes in d) and left lower lobe consolidation (red boxes in e). (f) Volume rendering of the chest CT scan obtained 3 days after admission shows the new areas of consolidation. They are reproduced from [15] Huang P, Liu T, Huang L, Liu H, Lei M, $\mathrm{Xu} \mathrm{W,} \mathrm{Hu} \mathrm{X,} \mathrm{Chen} \mathrm{J,} \mathrm{Liu} \mathrm{B.} \mathrm{Use} \mathrm{of} \mathrm{Chest} \mathrm{CT} \mathrm{in} \mathrm{Combination} \mathrm{with}$ Negative RT-PCR Assay for the 2019 Novel Coronavirus but High Clinical Suspicion. Radiology. 2020;295(1):22-23. doi:10.1148/radiol.2020200330

https://pubmed.ncbi.nlm.nih.gov/32049600/ under Copyright 2020 by the Radiological Society of North America, Inc.

This article is made available via the PMC Open Access Subset for unrestricted re-use and analyses in any form or by any means with acknowledgment of the source. These permissions are granted for the duration of the COVID-19 pandemic or until permits are revoked in writing. Upon expiration of these permissions, PMC is granted a perpetual license to make this article available via PMC and Europe PMC, consistent with existing copyright protections.

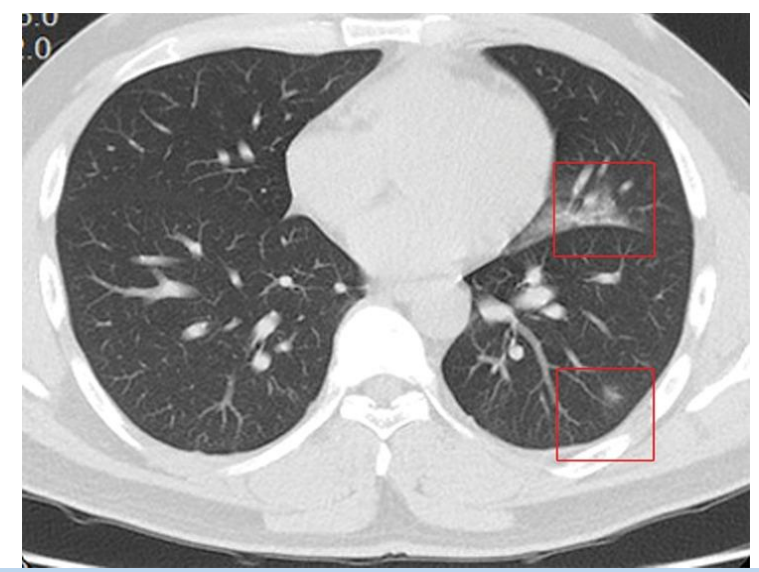

Figure 2c:

Images in a 36-year-old man with a 2-day history of fever, sore throat, and fatigue 5 days after visiting Wuhan, China, and a negative sputum real-time fluorescence polymerase chain reaction assay for the 2019 novel coronavirus. $(\mathrm{a}, \mathrm{b})$ Chest $\mathrm{CT}$ scans obtained at presentation show ground- glass opacities (red box) in the right upper lobe, and the lingular segment and the left lower lobe (b). (c) Volume rendering of a chest CT scan obtained at admission. (d, e) CT scanned obtained 3 days after admission show progression of ground-glass opacities to an atoll sign in the right upper lobe (red boxes in d) and left lower lobe consolidation (red boxes in e). (f) Volume rendering of the chest CT scan obtained 3 days after admission shows the new areas of consolidation. They are reproduced from [15] Huang P, Liu T, Huang L, Liu H, Lei M, Xu W, Hu X, Chen J, Liu B. Use of Chest CT in Combination with Negative RT-PCR Assay for the 2019 Novel Coronavirus but High Clinical Suspicion. Radiology. 2020;295(1):22-23. doi:10.1148/radiol.2020200330 https://pubmed.ncbi.nlm.nih.gov/32049600/ under Copyright 2020 by the Radiological Society of North America, Inc.

This article is made available via the PMC Open Access Subset for unrestricted re-use and analyses in any form or by any means with acknowledgment of the original source. These permissions are granted for the duration of the COVID-19 pandemic or until permits are revoked in writing. Upon expiration of these permissions, $\mathrm{PMC}$ is granted a perpetual license to make this article available via PMC and Europe PMC, consistent with existing copyright protections.

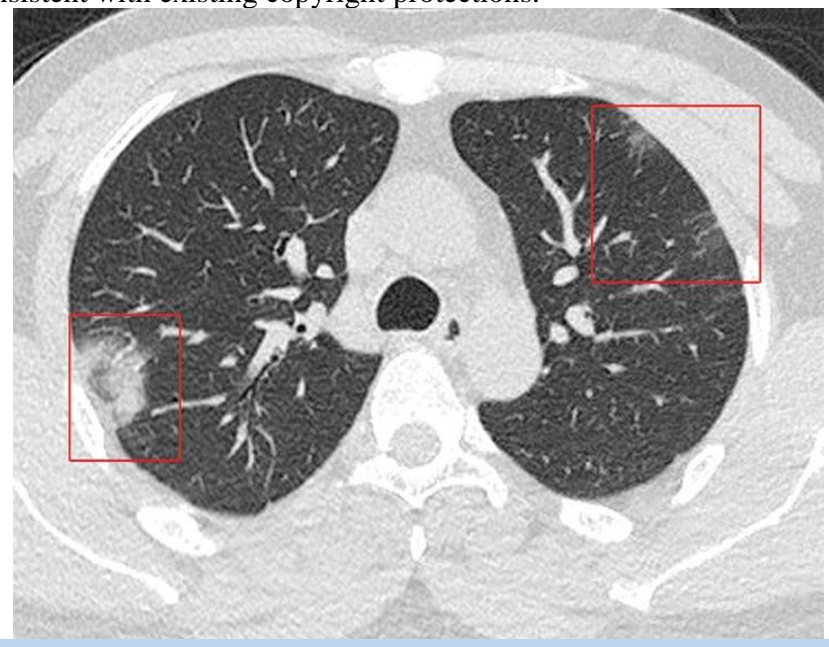

Figure 2 d.

Images in a 36-year-old man with a 2-day history of fever, sore throat, and fatigue 5 days after visiting Wuhan, China, and a negative sputum real-time fluorescence polymerase chain reaction assay for the 2019 novel coronavirus. $(\mathrm{a}, \mathrm{b}) \mathrm{Chest} \mathrm{CT}$ scans obtained at presentation show groundglass opacities (red box) in the right upper lobe, and the lingular segment and the left lower lobe (b). (c) Volume rendering of a chest CT scan obtained at admission. (d, e) CT scanned obtained 3 days after admission show progression of ground-glass opacities to an atoll sign in the right upper lobe (red boxes in d) and left lower lobe consolidation (red boxes in e). (f) Volume rendering of the chest CT scan obtained 3 days after admission shows the new areas of consolidation. They are reproduced from [15] Huang P, Liu T, Huang L, Liu H, Lei M, Xu W, Hu X, Chen J, Liu B. Use of Chest CT in Combination with Negative RT-PCR Assay for the 2019 Novel Coronavirus but High Clinical Suspicion. Radiology. 2020;295(1):22-23. doi:10.1148/radiol.2020200330 https://pubmed.ncbi.nlm.nih.gov/32049600/ under Copyright 2020 by the Radiological Society of North America, Inc.

This article is made available via the PMC Open Access Subset for unrestricted re-use and analyses in any form or by any means with acknowledgment of the original source. These permissions are granted for the duration of the COVID-19 pandemic or until permits are revoked in writing. Upon expiration of these permissions, PMC is granted a perpetual 
license to make this article available via PMC and Europe PMC, consistent with existing copyright protections.
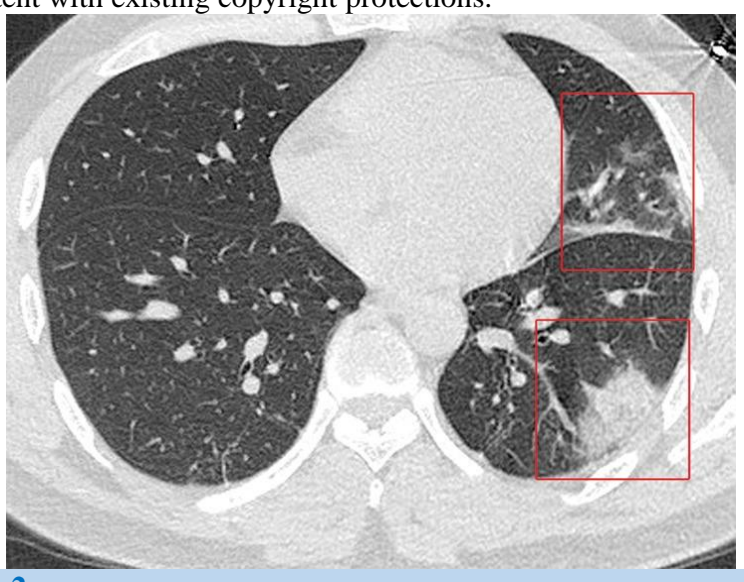

Figure 2 e.

Images in a 36-year-old man with a 2-day history of fever, sore throat, and fatigue 5 days after visiting Wuhan, China, and a negative sputum real-time fluorescence polymerase chain reaction assay for the 2019 novel coronavirus. (a, b) Chest CT scans obtained at presentation show groundglass opacities (red box) in the right upper lobe, and the lingular segment and the left lower lobe (b). (c) Volume rendering of a chest CT scan obtained at admission. (d, e) CT scanned obtained 3 days after admission show progression of ground-glass opacities to an atoll sign in the right upper lobe (red boxes in d) and left lower lobe consolidation (red boxes in e). (f) Volume rendering of the chest CT scan obtained 3 days after admission shows the new areas of consolidation. They are reproduced from [15] Huang P, Liu T, Huang L, Liu H, Lei M, Xu W, Hu X, Chen J, Liu B. Use of Chest CT in Combination with Negative RT-PCR Assay for the 2019 Novel Coronavirus but High Clinical Suspicion. Radiology. 2020;295(1):22-23.

doi:10.1148/radiol.2020200330 https://pubmed.ncbi.nlm.nih.gov/32049600/ under Copyright 2020 by the Radiological Society of North America, Inc.

This article is made available via the PMC Open Access Subset for unrestricted re-use and analyses in any form or by any means with acknowledgment of the original source. These permissions are granted for the duration of the COVID-19 pandemic or until permits are revoked in writing. Upon expiration of these permissions, PMC is granted a perpetual license to make this article available via PMC and Europe PMC, consistent with existing copyright protections.

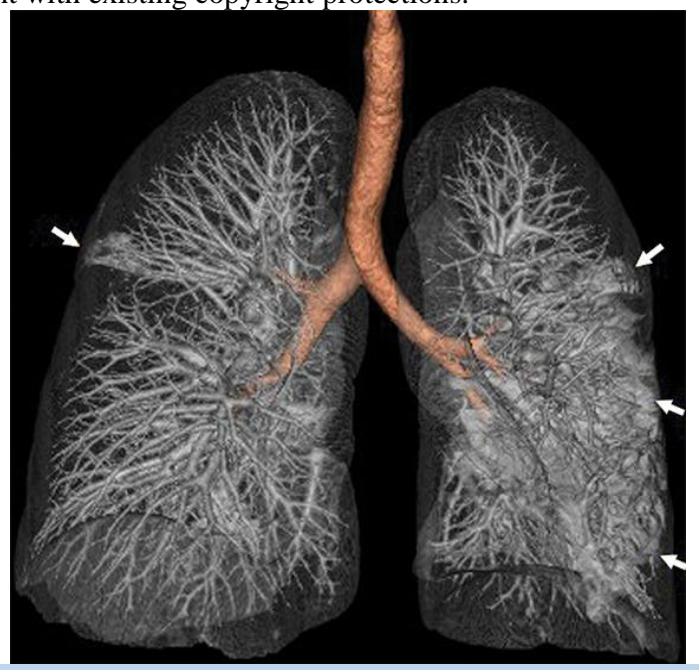

Figure 2f.
Images in a 36-year-old man with a 2-day history of fever, sore throat, and fatigue 5 days after visiting Wuhan, China, and a negative sputum real-time fluorescence polymerase chain reaction assay for the 2019 novel coronavirus. ( $a, b)$ Chest CT scans obtained at presentation show groundglass opacities (red box) in the right upper lobe, and the lingular segment and the left lower lobe (b). (c) Volume rendering of a chest CT scan obtained at admission. (d, e) CT scanned obtained 3 days after admission show progression of ground-glass opacities to an atoll sign in the right upper lobe (red boxes in d) and left lower lobe consolidation (red boxes in e). (f) Volume rendering of the chest CT scan obtained 3 days after admission shows the new areas of consolidation. They are reproduced from [15] Huang P, Liu T, Huang L, Liu H, Lei M, Xu W, Hu X, Chen J, Liu B. Use of Chest CT in Combination with Negative RT-PCR Assay for the 2019 Novel Coronavirus but High Clinical Suspicion. Radiology. 2020;295(1):22-23.

doi:10.1148/radiol.2020200330 https://pubmed.ncbi.nlm.nih.gov/32049600/ under Copyright 2020 by the Radiological Society of North America, Inc.

This article is made available via the PMC Open Access Subset for unrestricted re-use and analyses in any form or by any means with acknowledgment of the original source. These permissions are granted for the duration of the COVID-19 pandemic or until permits are revoked in writing. Upon expiration of these permissions, PMC is granted a perpetual license to make this article available via PMC and Europe PMC, consistent with existing copyright protections.

Pan et al. [16] undertook a study to observe the radiology imaging features of the novel coronavirus pneumonia. Pan et al. [16] enrolled sixty-three confirmed patients who had CVID-19 pneumonia from $30^{\text {th }}$ December 2019 to $31^{\text {st }}$ January 2020. The patients did undergo High-resolution CT (HRCT) of the thorax. Pan et al. [16] recorded the number of affected lobes of the lung, ground-glass nodules (GGO), patchy/punctate groundglass opacities, patchy consolidation, fibrous stripes as well as irregular solid nodules in each patient's computed tomography (CT) scan of the thorax image. Furthermore, pan et al. [16] performed a radiology imaging follow-up of the patients above. About the results, pan et al. [16] emphasized that they had collected the CT scan images of the 63 patients who were confirmed to have coronavirus pneumonia (Examples of some of the CT scan images have been illustrated in figure 4 and figure 5). Pan et al. [16] summated their findings as follows:

- $\quad$ The male to female $(\mathrm{M} / \mathrm{F})$ ratio of the patients was $33 / 30$.

- The mean age of the patients was $44.9+/-15.2$ years.

- The mean number of lobes that were affected by the disease was $3.3+/-1.8$.

- $\quad$ Nineteen patients that amounted to $30.2 \%$ of the patients had one affected lobe, five patients that amounted to $7.9 \%$ of the patients had two affected lobes of the lung, four patients that amounted to $6.3 \%$ had three affected lobes of the lung, seven patients that that amounted to $11.1 \%$ of the patients had four affected lobes of the lung, and 28 patients that amounted to $44.4 \%$ of the patients had five affected lobes of the lung.

- $\quad$ Fifty-four patients that amounted to $85.7 \%$ of the patients had patchy/punctate ground-glass opacities, 14 patients that amounted to $22.2 \%$ of the patients had GGO, 12 patients that amounted to $19.0 \%$ of the patients had patchy consolidation, 11 patients that amounted to $17.5 \%$ of the patients had fibrous stripes, and 8 patients that amounted to $12.7 \%$ of the patients did have irregular solid nodules.

- Fifty-four patients that amounted to $85.7 \%$ of the patients progressed that included single GGO that increased, enlarged as well as consolidated; fibrous stripe that enlarged, while solid nodules did increase and enlarged.

Pan et al. [16] made the ensuing conclusions: 
- Radiology imaging changes that occur in novel viral pneumonia tends to be rapid.

- The presentations of the novel coronavirus pneumonia are diverse.

- $\quad$ Radiology imaging changes of typical viral pneumonia and some specific imaging features had been observed.

- Given the findings above, there is a need for the strengthening of the recognition of radiology image changes to help clinicians to quickly and accurately diagnose COVID-19 pneumonia.

Considering that only 63 patients were enrolled in the study of Pan et al. [16], it would be argued that perhaps there are other radiology imaging features that some cases of COVID-Pneumonia would demonstrate. For this reason, clinicians should be encouraged to report instances of COVID-19 pneumonia they have managed as well as lessons learned from the management of COVID-19. The radiology imaging features that had been found by Pan et al. [16] are non-specific radiology image findings. Given this, we have considered various types of radiology image features that would tend to simulate some of the non-specific image features of COVID-19 pneumonia. Unfortunately, the radiology images related to the article of Pan et al. [16] are not easily available to be reproduced in a new article even though there is a clearance center with guidelines for obtaining permission to re-use images in the report. Instead, we have included images from the archives of one of the authors to illustrate possible radiology images that should alert clinicians to the possibility of COVID-19 pneumonia. In the latter part of the article, we have discussed various other radiology images that could simulate COVID-19 infection and for which necessary tests would need to be undertaken to confirm or negate COVID-19 pneumonia.

Dai et al. [20] stated that since the beginning of 2020, coronavirus disease 2019 (COVID-19) has spread throughout China. This study has explained the findings from lung computed tomography images of some patients with COVID-19. They were treated in a medical institution and has discussed the difference between COVID-19 and other lung diseases. Dai et al. [20] reported some cases of COVID19 as follows:

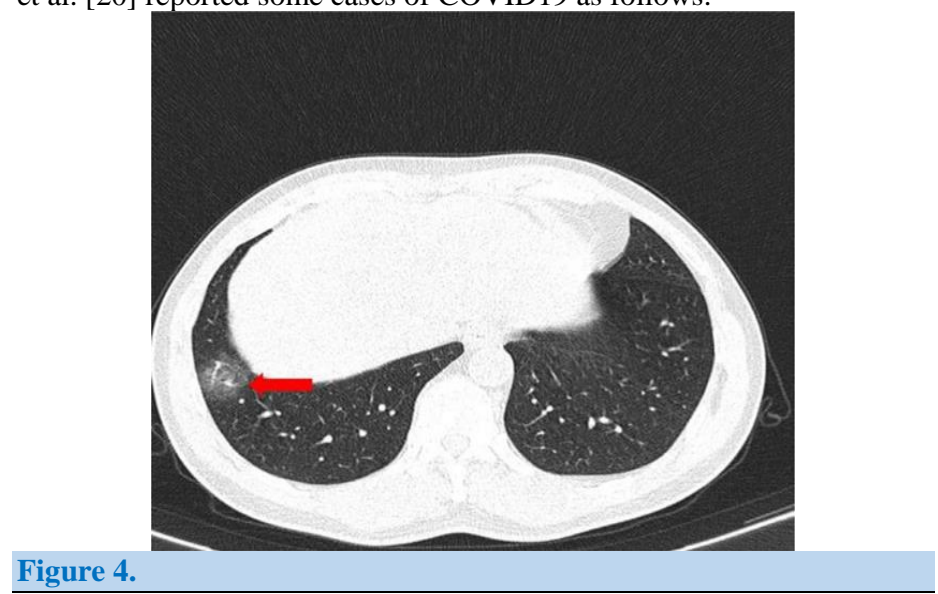

\section{Case 1}

A 52-year-old man who had been working in Wuhan, in China, had arrived in Shenzhen in China, on the $22^{\text {nd }}$ of January 2020 . He requested treatment because he had a half a day symptom of fever that was associated with nasal congestion, as well as a headache. Still, he did not have any rhinorrhoea, pharyngula, cough, expectoration, or diarrhea. The results of his laboratory blood tests demonstrated a low lymphocyte count of $0.58 \times 10^{9} / 1$, a high neutrophil percentage of $81.3 \%$, a high procalcitonin concentration of $0.130 \mathrm{ng} / \mathrm{mL}$, a high-sensitivity C-reactive protein (hs-CRP) concentration of $7.80 \mathrm{mg} / \mathrm{l}$, a normal white blood cell (WBC) count of $6.62 \times 10^{9} / \mathrm{L}$, as well as normal concentrations of creatinine kinase-myocardial band, cardiac troponin 1 , as well as myoglobin. He had an unenhanced CT scan of the thorax, which demonstrated patchy pure ground-glass opacities (GGOs) within the lateral basal segment of his right lower lobe lung as well as vascular dilatation inside the lesion (see figure 3). He was suspected of having early staged COVID 19 pneumonia. The NAAT result that was obtained by utilization of RT-PCR identification of SARS-CoV-2 from his respiratory tract specimens was reported to be positive, and hence he was diagnosed as having COVID-19.

Figure 3. A 52-year old male: Computed tomography scan revealed patchy pure ground-glass opacities (red arrow) at the lateral basal segment of the right lower lobe and vascular dilation inside the lesion. Reproduced from: [20] Dai W C, Zhang H W, Yu J, Xu H J, Chen H, Luo SP, Zhang $\mathrm{H}$, Liang L H, Wu X L, Lei Y, Lin F. CT Imaging and Differential Diagnosis of COVID-19. Can Assoc Radiol 2020 May; 71(2): 195 $200 \quad$ DOI: $\quad \mathbf{1 0 . 1 1 7 7 / 0 8 4 6 5 3 7 1 2 0 9 1 3 0 3 3 .}$ https://pubmed.ncbi.nlm.nih.gov/32129670/

Under Copyright (C) The Author(s) 2020

This article is distributed under the terms of the Creative Commons Attribution $4.0 \quad$ License (http://www.creativecommons.org/licenses/by/4.0/), which permits any use, reproduction, and distribution of the work without further permission provided the original work is attributed as specified on the SAGE and Open Access pages (https://us.sagepub.com/en-us/nam/open-access-atsage).

\section{Case 2}

A 63-years-old man who had taken a high-speed train from Wuhan to Beijing on the $10^{\text {th }}$ of January 2020 did arrive in Shenzhen by plane from Beijing on the $26^{\text {th }}$ of January 2020 . He was admitted to hospital, given him having a fever over the preceding 5 days pursuant to catching a cold, and his highest body temperature was $37.7^{\circ} \mathrm{C}$. He also had an occasional cough as well as myalgia, but this was not associated with expectoration or dyspnoea. The results of his laboratory tests revealed elevated hs-CRP of $33.57 \mathrm{mg} / \mathrm{L}$, normal procalcitonin level of $0.072 \mathrm{ng} / \mathrm{mL}$, a normal neutrophil count of $2.50 \times 10^{9} / \mathrm{L}$, a normal lymphocyte count of the level of $1.11 \times 10^{9} / \mathrm{L}$, a normal white blood cell (WBC) count of $3.99 \times 10^{9} / \mathrm{L}$, as well as a normal lymphocyte percentage of $27.8 \%$. He had an unenhanced computed tomography (CT) scan of the thorax which revealed multiple pure ground-glass opacities (GGOs) within the right upper lobe of his lung, distribution of lesions within the sub-pleural area and the periphery of the lung, a "crazy-paving" pattern, as well as interlobular septal thickening (see figure 4). Based upon the computed tomography (CT) scan of thorax features, the patient was provisionally suspected to be affected by advanced-staged COVID19. The result of his NAAT test that was obtained by means of the undertaking of RT-PCR detection of SARs-2 from the patient's blood specimens was positive, which confirmed the diagnosis of COVID-19.

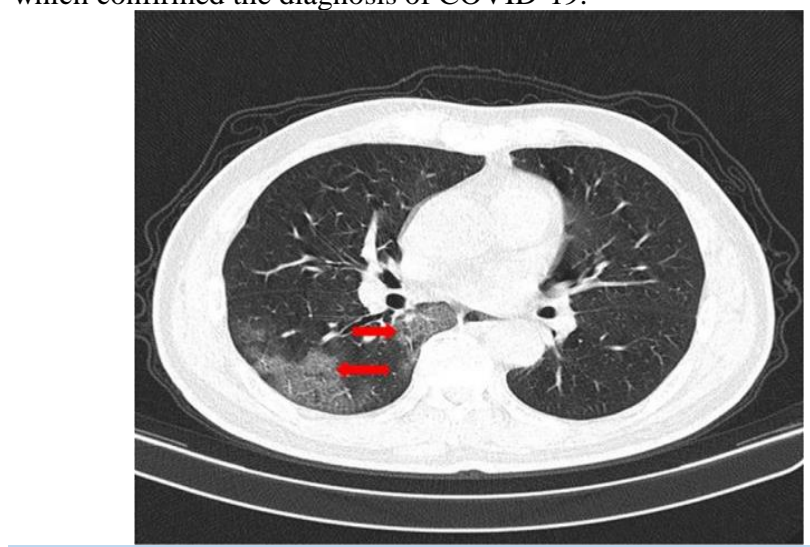

Figure 4. 
A 63-year-old male: High-resolution computed tomography scan showed multiple pure ground-glass opacities in the right lobe, distribution of lesions in the subpleural area, and lung periphery, a "crazy-paving" pattern, and interlobular septal thickening (red arrow). Reproduced from: [20] Dai W C, Zhang H W, Yu J, Xu H J, Chen H, Luo SP, Zhang H, Liang $\mathrm{L} \mathrm{H}, \mathrm{Wu} X \mathrm{~L}$, Lei $\mathrm{Y}$, Lin F. CT Imaging and Differential Diagnosis of COVID-19. Can Assoc Radiol 2020 May; 71(2): 195 $200 \quad$ DOI: $\quad \mathbf{1 0 . 1 1 7 7 / 0 8 4 6 5 3 7 1 2 0 9 1 3 0 3 3 .}$ https://pubmed.ncbi.nlm.nih.gov/32129670/

Under Copyright (C) The Author(s) 2020

This article is distributed under the terms of the Creative Commons Attribution $4.0 \quad$ License (http://www.creativecommons.org/licenses/by/4.0/), which permits any use, reproduction, and distribution of the work without further permission provided the original work is attributed as specified on the SAGE and Open Access pages (https://us.sagepub.com/en-us/nam/open-access-atsage).

\section{Case 3}

Case 3 involved a 48-years-old man who had been quarantined at home after he had driven himself from Wuhan to Shenzhen on the $24^{\text {th }}$ of January 2020. He was admitted to hospital because of having pharyngula over the preceding 4 days as well as a fever that had lasted over the previous 1 day. His temperature upon his admission was $38.5^{\circ} \mathrm{C}$. He did not have any evidence or history of cough, expectoration, headaches, myalgia, nasal congestion, rhinorrhea, or dyspnoea. The results of his laboratory blood test, demonstrated elevated serum hs-CRP level of 73.99 $\mathrm{mg} / \mathrm{mL}$, increased procalcitonin concentration level of $0.100 \mathrm{ng} / \mathrm{mL}$, a decreased lymphocyte count level of $0.92 \times 10^{9} / \mathrm{L}$, and a normal white blood cell (WBC) count of $5.54 \times 10^{9} / \mathrm{L}$. He had unenhanced computed tomography (CT) scan of the thorax that demonstrated multiple groundglass opacities (GGOs) within both of his lungs, inter-septal thickening, as well as a "crazy-paving pattern" (see figure 5). Based upon the computed tomography (CT) scan of the thorax features of the patient, he was provisionally diagnosed as having advanced-stage COVID-19. The result of his NAAT, which was obtained by the undertaking of RT-PCR detection test for SARS-CoV-2 from his pharyngeal swabs, was positive for COVID-19, and he was diagnosed as having COVID-19.

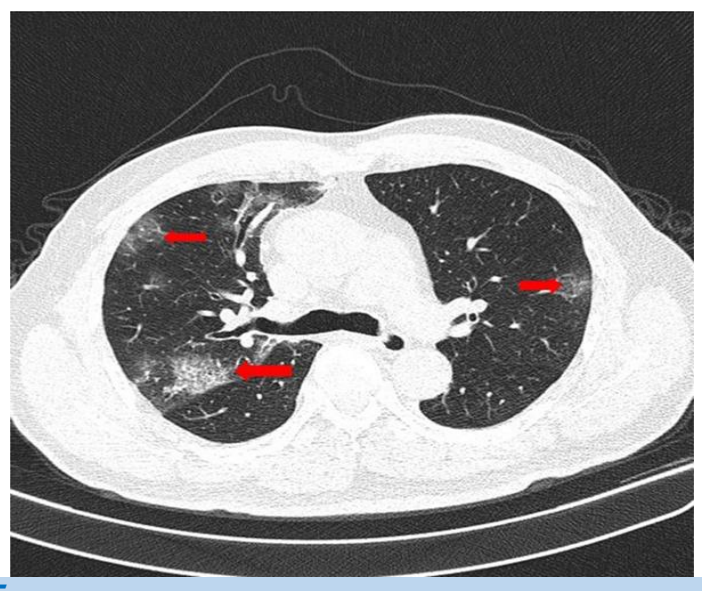

Figure 5.

A 48-year-old male: High-resolution computed tomography scan showed multiple ground-glass opacities in numerous lobes of both lungs, interlobular septal thickening, and a crazy-paving pattern (red arrow). Reproduced from: [20] Dai W C, Zhang H W, Yu J, Xu H J, Chen H, Luo SP, Zhang $\mathrm{H}$, Liang $\mathrm{L} \mathrm{H}, \mathrm{Wu} \mathrm{X} \mathrm{L}$, Lei Y, Lin F. CT Imaging and Differential Diagnosis of COVID-19. Can Assoc Radiol 2020 May;
71(2): $195 \quad-200 \quad$ DOI: $10.1177 / 0846537120913033$. https://pubmed.ncbi.nlm.nih.gov/32129670/

Under Copyright (C) The Author(s) 2020

This article is distributed under the terms of the Creative Commons Attribution $4.0 \quad$ License (http://www.creativecommons.org/licenses/by/4.0/), which permits any use, reproduction, and distribution of the work without further permission provided the original work is attributed as specified on the SAGE and Open Access pages (https://us.sagepub.com/en-us/nam/open-access-atsage).

\section{Case 4}

This case involved a 47-year-old male who was a resident of Shenzhen and who had been within close contact of less than 2 meters recently with a driver from Wuhan. He had had a fever for the preceding 4 days even though he had used non-prescription medications, because of the sought treatment within the hospital His body temperature was $40^{\circ} \mathrm{C}$ as well as he had diarrhea twice per day. Still, he did not have any cough, expectoration, myalgia, dysuria, or dyspnea. The results of his laboratory blood tests revealed a low percentage of lymphocytes of $14.4 \%$, a low lymphocyte count of $0.90 \times 10^{9} / \mathrm{L}$, a high hs-CRP concentration of 136.51 $\mathrm{mg} / \mathrm{L}$, a high procalcitonin concentration of $0.180 \mathrm{ng} / \mathrm{mL}$, a normal neutrophil percentage of $72.2 \%$ and a normal white blood cell (WBC) count of $6.27 \times 10^{9} / \mathrm{L}$. He had un-enhanced computed tomography (CT) scan of the thorax that demonstrated patchy consolidations upon his left upper lobe lung and partially consolidated lung tissue, ground-glass opacities (GGOs) on edge, and air bronchograms within the lesion (see figure 6). The result of his NAAT that was based upon RT-PCR detection of SARS-CoV-2 from a specimen of his blood was positive for COVID19 , and therefore a final diagnosis of COVID19 was made.

A lesson that has been learned from those above 4 reported cases is the fact that instances of advanced-stage or well-established cases of COVID would tend to demonstrate upon radiology imaging of CT scan of thorax evidence of various opacities within the lung including ground-glass opacities pari passu positive COVID-19 test results and given this within the era of COVID19 pandemic any individual who has respiratory tract symptoms which are shown upon radiology imaging to have pulmonary metastasis should be made to undergo COVID-19 to clarify the situation.

\section{Figure 6.}

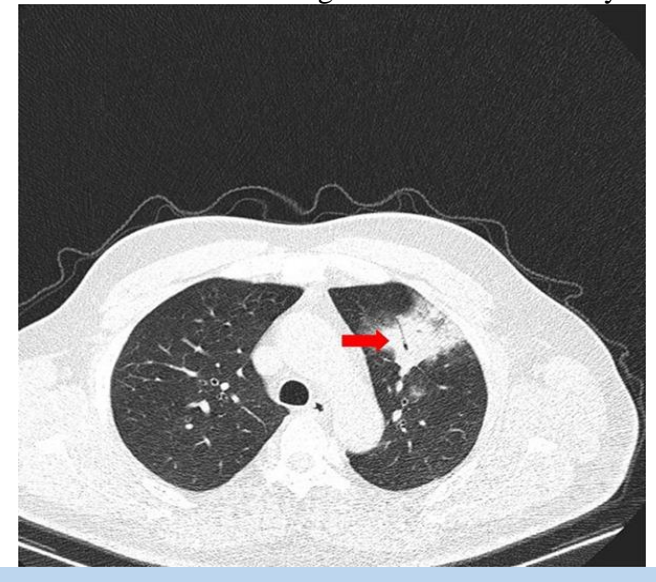

A 47-year-old male: High-resolution computed tomography scan showed patchy consolidations on the left upper lobe, partially consolidated lung tissue, ground-glass opacities on edge, and air bronchograms in the lesion (red arrow). Reproduced from: [20] Dai W C, Zhang H W, Yu J, Xu H J, Chen H, Luo SP, Zhang H, Liang L H, Wu X L, Lei Y, Lin F. CT Imaging and Differential Diagnosis of COVID-19. Can Assoc Radiol 2020 
May; 71(2): $195 \quad-\quad 200$ DOI: $10.1177 / 0846537120913033$. https://pubmed.ncbi.nlm.nih.gov/32129670/

Under Copyright (C) The Author(s) 2020

This article is distributed under the terms of the Creative Commons Attribution $4.0 \quad$ License (http://www.creativecommons.org/licenses/by/4.0/), which permits any use, reproduction, and distribution of the work without further permission provided the original work is attributed as specified on the SAGE and Open Access pages (https://us.sagepub.com/en-us/nam/open-access-atsage).

Dai et al. [20] made the ensuing iterations:

- Computed tomography (CT) scan imaging tends to be associated with the limitation in that some radiology imaging signs of COVID-19 tend to simulate those of other pulmonary diseases.

- $\quad$ The ensuing two (2) patients (cases 5 and 6 in our article and cases 1 and 2 in their original article did have suspected COVID-19 within their institution and were paid special attention based upon a provisional suspicion of them having COVID-19; nevertheless, they were finally diagnosed as having other disease and not COVID-19:

\section{Case 5 (Case 1 in Dai et al. [20])}

A 60-year-old woman who did not have any recent history of exposure to the COVID-19 pandemic area was admitted to hospital because she had been coughing for 10 days. She also had chest pain and had been wheezing over the preceding 3 days, as well as she had been producing yellow sputum. She had been taking cephalosporin antibiotics by herself before seeking treatment within the hospital, but her symptoms had not improved. She did not have any fever, headache, rhinorrhea, nausea, or vomiting. She did have a medical history of rheumatoid arthritis for more than 10 years. The results of her laboratory blood test, demonstrated a normal white blood cell (WBC) count of $4.96 \times 10^{9} / 1$, a normal neutrophil percentage of $48.6 \%$, a normal procalcitonin level, as well as a normal lymphocyte count status. He had a plain chest x-ray, which demonstrated extensive patchy exudates as well as consolidation upon both of his lungs, faint ground-glass opacity (GGOs) upon the edge, as well as interlobular septal thickening (see figure 7). The result of her NAAT that was obtained using the RT-PCR detection test for SARS-CoV-2 from her respiratory tract specimens was negative for COVID-19. The results of her additional laboratory blood tests revealed high hs-CRP of $40.20 \mathrm{mg} / \mathrm{L}$, high Ddimer of $2.22 \mathrm{mg} / \mathrm{L}$, high immunoglobulin G level of $19.3 \mathrm{~g} / \mathrm{L}$, as well as positive rheumatoid factor. Her final diagnosis was rheumatoid pneumonia. The lesson learned from this case report is that not all cases that are provisionally diagnosed as possible COVID19 are COVID19.

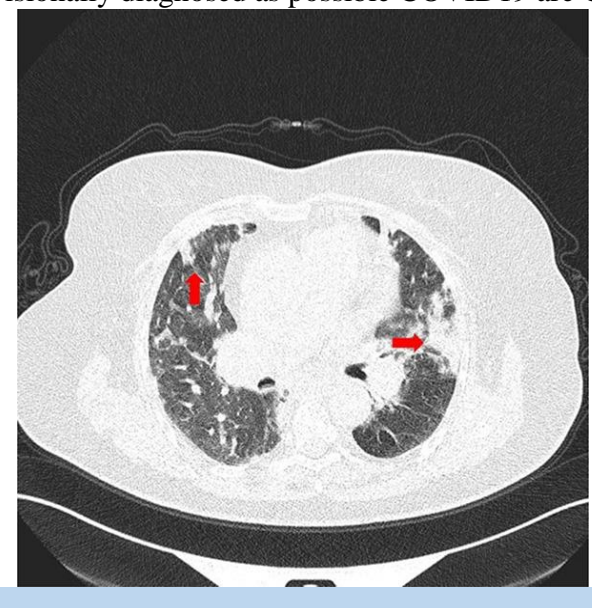

Figure 7.

Auctores Publishing - Volume 1(3)-019 www.auctoresonline.org ISSN: 2692-9406
A 60-year-old female: High-resolution chest computed tomography scan revealed extensive patchy exudates and consolidation of both lungs, faint ground-glass opacities on edge, and the interlobular septal thickening (red arrow). Reproduced from: [20] Dai W C, Zhang H W, Yu J, Xu H J, Chen H, Luo SP, Zhang H, Liang L H, Wu X L, Lei Y, Lin F. CT Imaging and Differential Diagnosis of COVID-19. Can Assoc Radiol 2020 May; 71(2): $195 \quad-200$ DOI: $10.1177 / 0846537120913033$. https://pubmed.ncbi.nlm.nih.gov/32129670/

Under Copyright (C) The Author(s) 2020

This article is distributed under the terms of the Creative Commons Attribution $4.0 \quad$ License (http://www.creativecommons.org/licenses/by/4.0/), which permits any use, reproduction, and distribution of the work without further permission provided the original work is attributed as specified on the SAGE and Open Access pages (https://us.sagepub.com/en-us/nam/open-access-atsage).

\section{Case 6 (Case 2 in Dai et al. [20]).}

A 58-year-old woman from Henan and who had recently passed through Wuhan had manifested with recurrent chest pain for 1 month. She was admitted to hospital 2 hours under aggravation of her chest pain. She did not have any fever, cough, dizziness, or headache, but she did have a history of hypertension for longer than 10 years. She had a computed tomography (CT) scan of thorax, which demonstrated extensive groundglass opacities (GGOs) within both of her lungs, which was mainly distributed along her hila, interlobular septal thickening, as well as interlobar pleural thickening (see figure 8). She had a computed tomography (CT) scan of the thorax, and based upon the features that were demonstrated by the CT scan of thorax, the possibility of COVID19 could not be excluded. The result of her NAAT test that was obtained using RTPCR detection of SARS-CoV-2 from specimens of her tract was noted to be negative. The results of her laboratory blood test did not reveal any abnormality in her D-dimer concentration, white blood cell count (WBC), or hs-CRP concentration. She had echocardiography, which demonstrated low-amplitude left ventricular wall motion as well as low diastolic function. She had electrocardiography, which showed ST elevation in lead aVR of $0.05 \mathrm{mV}$ as well as multi-lead ST depression of 0.1 to 0.2 $\mathrm{mV}$. She had digital subtraction angiography, which did suggest occlusion of her left anterior descending coronary artery. A final diagnosis of heart failure and pulmonary edema that had been caused by coronary heart disease and acute myocardial infarction was made. The lesson learned from this case report is that cardiopulmonary conditions, including heart failure related to coronary artery disease, myocardial infarction, and pulmonary edema, can mimic COVID19 pneumonia.

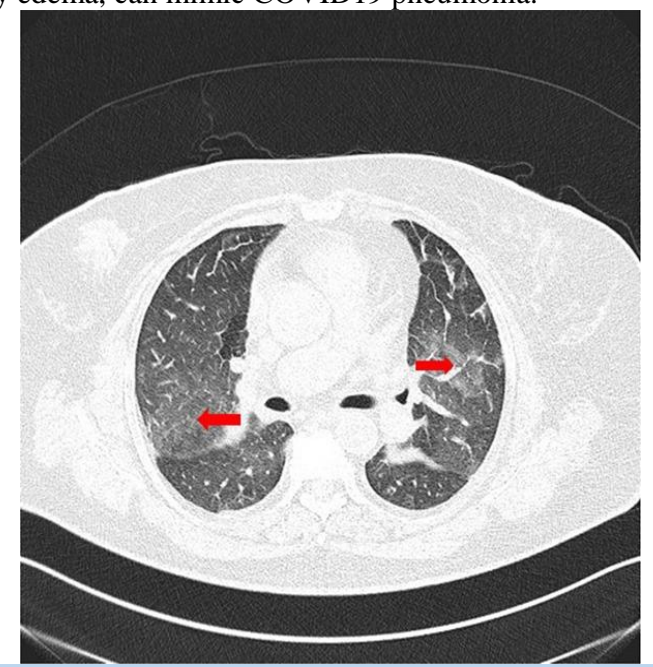

Figure 8. 
A 58-year-old female: High-resolution chest CT showed extensive GGOs in both lungs, which were mainly distributed along the hila, interlobular septal thickening, and interlobar pleural thickening (red arrow). Reproduced from: [20] Dai W C, Zhang H W, Yu J, Xu H J, Chen H, Luo SP, Zhang H, Liang L H, Wu X L, Lei Y, Lin F. CT Imaging and Differential Diagnosis of COVID-19. Can Assoc Radiol 2020 May; 71(2): $195 \quad-200$ DOI: $10.1177 / 0846537120913033$. https://pubmed.ncbi.nlm.nih.gov/32129670/

Under Copyright (C) The Author(s) 2020

This article is distributed under the terms of the Creative Commons Attribution $4.0 \quad$ License (http://www.creativecommons.org/licenses/by/4.0/), which permits any use, reproduction, and distribution of the work without further permission provided the original work is attributed as specified on the SAGE and Open Access pages (https://us.sagepub.com/en-us/nam/open-access-atsage).

$\mathrm{Xu}$ et al. [21] reviewed data related to all patients who had a laboratory test identified cases of SARS-CoV-2 infection that were confirmed based upon real-time polymerase chain reaction (PCR) between $23^{\text {rd }}$ January and $4^{\text {th }}$ February 2020 within a designated hospital called Guangzhou Eighth People's hospital. Ninety (90) patients, which included 39 males and 51 females whose ages had ranged between 18 years and 86 years and whose median age was 50 years, were included in the review study. Xu et al. [21] emphasized that all of the patients who had SARS-CoV-2 infections had undergone non-contrast-enhanced computed tomography (CT) scan. Xu et al. [21] did analyze the clinical features of the patients, and they also analyzed the distribution characteristics, the pattern of the disease, the morphology of the disease, as well as the accompanying presentations of the lung lesions. They evaluated after 1 day to 6 days with a mean time of after 3.5 days; they assessed the follow-up computed tomography (CT) scan of thorax images of the patients to ascertain the radiological evolution of the disease. $\mathrm{Xu}$ et al. [21] summated their findings as follows:

- $\quad$ Most of the COVID-19 infected patients did have a history of exposure to the disease in Wuhan or exposure to infected patients, and they mostly manifested with fever and cough.

- More than half of the cohort of patients manifested with bilateral, multi-focal lung lesions, with peripheral distribution, and 53 patients that amounted to $59 \%$ of the patients did have more than two lobes of the lung involved.

- $\quad$ Concerning all of the patients that had been enrolled within the study, COVID-19 pneumonia did manifest with ground-glass opacities in 65 patients that amounted to $72 \%$ of the patients, consolidation in 12 patients that amounted to $13 \%$ of the patients, crazy paving pattern in 11 patients that amounted to $12 \%$ of the patients, inter-lobular thickening in 33 patients that amounted to $37 \%$ of the patients, adjacent pleural thickening concerning 50 patients that amounted to $56 \%$ of the patients, as well as linear opacities combined in 55 patients that amounted to $61 \%$ of the patients.

- Pleural effusion, pericardial effusion. As well as lymph node enlargement tended to be uncommon findings.

- $\quad$ Furthermore, baseline computed tomography (CT) scans of the thorax did not reveal any abnormalities concerning 21 of the patients that amounted to $23 \%$ of the patients; nevertheless, 3 of the patients did manifest with bilateral ground-glass opacities upon the second computed tomography (CT) scan according to 3 to 4 days.

Xu et al. [21] made the ensuing concluding stipulations:

- The SARS-CoV-2 infection could be confirmed based upon the history of the patient, the clinical presentations, the radiology imaging features, as well as the results of the laboratory tests.
- Computed tomography (CT) scan of thorax examination does play a pivotal role concerning the initial diagnosis of the novel coronavirus pneumonia.

- Multiple patchy ground-glass opacities in bilateral multiple lobular with the peripheral distribution of the lung are typical computed tomography (CT) scan of thorax, imaging features of the COVID-19 pneumonia infection.

Zhou et al. [22] undertook a retrospective observational study that was based upon data that had been collected between $19^{\text {th }}$ January 2020 and $15^{\text {th }}$ February 2020 on patients who had been diagnosed as having COVID-19 for which they had been treated. They reviewed the clinic workflow with the utilization of computed tomography (CT) scan of the thorax and RT-PCR assay to screen patients who are suspected of having COVID-19 pneumonia. The clinical data of the patients were evaluated, and the patients had been classified into (a) mild group, (b) common group, (c) severe group, and (d) critical group. They evaluated the computed tomography (CT) scan of the thorax features of each patient, and they did apply a CT scan scoring system to grade the involvement of the lung by the COVID-19. Zhou et al. [22] summarized the results as follows:

- $\quad$ Out of the 98 patients who had been enrolled in the study, 1, 29, 51, and 17 patients were classified clinically into mild, common, severe, and critical groups, respectively.

- Eighty-three patients that amounted to $84.7 \%$ of the patients had CT scan features that included: ground-glass opacity (GGO) that amounted to $84.7 \%$ of patients, 76 patients that amounted to $77.5 \%$ of the patients had consolidation within their lungs, and 18 patients that amounted to $18.4 \%$ had features of crazy-paving pattern on their computed tomography (CT) scan of the thorax.

- $\quad$ Based upon the computed tomography (CT) scan scoring, 2, 35, 55 , and 6 patients had been categorized into grade 0 , grade 1 , grade 2, and grade 3 respectively which was found to be significantly consistent with the clinical classification of the disease (kappa $=0.638, \mathrm{P} .<0.05$ ).

- Twenty-nine of the patients were admitted from the fever clinic with an average interval of 1.2 days between the CT scan examination and the onset of the symptom. Still, the interval had ranged between 0 and 4 days.

- Three of the patients did have negative initial RT-PCR results, but they had abnormalities that were demonstrated on their initial computed tomography (CT) scans of the thorax.

Zhou et al. [22] made the ensuing conclusions:

- Peripheral lung distributed ground-glass opacity (GGO) and consolidation in the absence of sub-pleural sparing, is the most frequent presentations upon computed tomography (CT) scan of the thorax of COVID-19 pneumonia.

- Abnormalities upon computed tomography (CT) scan of thorax could occur in an early stage of COVID-19, even when the result of RT-PCR assay is negative, which could help concerning the early recognition and diagnosis of COVID-19 pneumonia.

De Farias et al. [23] summarized the role of chest x-ray in COVID-19 infection as follows:

- The undertaking of a chest $\mathrm{x}$-ray in the assessment of patients who have or are suspected of having COVID-19 is a quick as well as the easy method that is frequently requested because of its wide availability as well as the low cost of the procedure.

- It has been stated that the advent utilization of portable devices has enabled its use within intensive care departments and within field hospitals. [24]. 
- It has been pointed out that the utilization of chest X-rays in assessing individuals with COVID-19 pneumonia has its limitations related to its low sensitivity, which has been estimated at $25 \%$, especially about initial cases of COVID-19 [25]. Based on the reason above, it has been emphasized that chest x-ray should not be regarded as a method of screening. [24].

- Utilization of chest x-ray has been recommended for a selected population of patients, including patients who are hospitalized to the progression of their disease and to assess the associated complications of COVID-19 pneumonia, such as ventilatorassociated pneumonia, pleural effusion, or pneumonia. [26]

- The main chest radiograph findings that had been documented in cases of COVID-19 include lung opacities that tend to be associated with bilateral distribution and predominance of the opacities within the periphery of the lung as well as within the lower pulmonary fields. [27]

- It has been pointed out that information that obtained related to the chest x-ray features of COVID-19 could either represent under detection of the disease or underestimation of the disease in comparison with the utilization of computed tomography (CT) scan of the thorax, and this does limit its capability about the monitoring of the disease. [25]

De Farias et al. [23] also made summations related to the undertaking of computed tomography scan of the thorax in cases of COVID-19 as follows:

- The undertaking of computed tomography scan of the thorax should be done about patients who have been admitted to hospital as well as symptomatic individuals who have worsening of their clinical conditions and about patients who have associated co-morbidities. [24] [28] [29] [30]. [10] [31] About these COVID-19 patients, CT scan of the thorax, tends to be indicated mainly to ascertain the extent of the COVID-19 pneumonia disease as well as to identify complications that emanate from the disease including pulmonary thromboembolism or any overlapping bacterial infection as well to assess the pulmonary features about their assessment for differential diagnoses. [29].

- About the comparison of CT scan of thorax with the reference diagnostic test of RT-PCR, a CT scan is stated to have a high sensitivity of $97 \%$. Still, CT scan does have low specificity of $25 \%$ [32], and given this CT scan of the thorax cannot replace utilization of RT-PCR. [24].

- The CT scan tomography features that are considered typical of COVID-19 include ground-glass opacities (GGOs), consolidations and crazy pattern with bilateral and multi-focal distribution, and a peripheral as well as posterior predominance. [33]. [34]. [35]. Nevertheless, it has been pointed out that the findings above are not exclusively found in cases of COVID19. Still, they can also be found in cases of other viral pneumonia, connective tissue lung disease, as well as druginduced pulmonary disease. [30] Given this, utilization of clinical data, as well as temporal correlation of the data with radiology image features of the patients, are essential, as well as the correlation of the clinical conditions of the patients. With the previous examination, findings would be helpful. Furthermore, the CT scan of the thorax of patients who have COVID-19 could also demonstrate signs of organizing pneumonia, reversed halo sign, reticular pattern, sub-pleural curvilinear lines, parenchymal bands, pseudo-cavities, nodules, and at times, configuring the halo sign. [36] [37] [38]. It has been pointed out that airway centered disease including thickening of bronchial wall, centrilobular and tree in bud opacities, pleural effusion, and lymph node enlargement tend not to be encountered frequently during the initial manifestation of COVID-19 infection. [36]

- The CT scan of thorax features that are seen does vary based upon the number of days from the onset of the symptoms. GGOs tend to be more commonly seen within the first few days of the onset of the symptom, and GGOs could evolve to consolidation, which reach a peak in pulmonary opacities between the $9^{\text {th }}$ day and the $13^{\text {th }}$ day under the onset of symptoms. [29]. [15] [34].

- It has been pointed out that dissociation between the results of the laboratory tests and the computed tomography scan of thorax findings could be found in patients who have positive RT-PCR results and the absence of radiology image findings and those who have extensive CT scan of thorax findings and negative RT-PCR result. [24] [39].

De Farias et al. [23] also said the ensuing:

- During a pandemic, a more sensitive interpretation of computed tomography of thorax findings that are related to COVID-19 had become necessary to aid the management of patients. Given this, some authors [30] [40] had suggested the elaboration of structured reports. This type of reporting does offer advantages concerning the context of high demand examinations as well as the need for quick decisions to be made.

- Concerning the radiologist, it does help in the elaboration of the report of the radiology images; it does decrease the reporting time as well as the variability of the reporting, as well as it does reduce uncertainty about the reporting of the findings. About the referring medical officer/physician, it does improve the understanding of the radiology image findings and the quality of the information that had been transmitted by the radiologist to the reporting physician, which does allow for better clinical management. [41].

- Out of the available classifications, the proposal of the consensus of specialists of the Radiological Society of North America [30] has been widely utilized.

- The Consensus of Specialists of the Radiological Society of North America [30] had proposed four groups of computed tomography (CT) scan findings/features that include: (a) typical, (b) indeterminate, (c) atypical, and (d) negative for COVID-19 (see figure 4) and had suggested ways to report them. De Farias et al. [23] adopted this classification with small reporting language changes, as illustrated in table 1.

De Farias et al. [23] made the ensuing conclusions:

- The undertaking of radiology imaging should be wisely indicated about the evaluation of patients who have COVID-19 to avoid unnecessary exposure of other patients and health care workers as well as to rationalize the utilization of personal protective equipment. The undertaking of radiology imaging should be reserved for individual patients who have moderate to severe respiratory tract symptoms, risk of progression of COVID-19 pneumonia due to the presence of concomitant comorbidities, or worsening of the respiratory tract medical condition. The undertaking of radiology imaging should not be indicated about patients who do not have any symptoms or those who have mild symptoms. Within environments that have limited COVID-19 testing resources, radiology imaging could eventually be indicated as a triage method about suspected patients with high pre-test probability.

- Computed tomography (CT) scan of the thorax is the main radiology imaging option that is utilized about the evaluation of individuals for COVID-19 pneumonia. Typical radiology imaging findings in cases of COVID-19 do include groundglass opacities (GGOs) with or without consolidations, crazy- 
paving pattern with bilateral and multifocal distribution, peripheral and posterior predominance, multi-focal GGOs of rounded morphology, as well as reversed halo sign. Furthermore, the undertaking of computed tomography (CT) scan of thorax could be useful about the evaluation of the extent of pulmonary disease, presence of complications, as well as the differential diagnosis of the disease. Despite the finding of typical computed tomography (CT) findings that tend to be demonstrated in COVID-19, RT-PCR does remain the gold standard for the diagnosis of COVID-19.

- $\quad$ Structured computed tomography (CT) scan of the thorax report does standardize the radiology imaging finding features. It does optimize communication with the referring medical doctor, and a CT scan of the thorax has been a useful tool within the COVID-19 pandemic scenario.

Zheng et al. [42] made the ensuing summations related to COVID-19 infection:

- COVID-19 tends to be transmitted mainly using respiratory tract droplets as well as close contacts that do cause a cluster of infections.

- The symptoms of COVID-19, tend to be predominated by fever, fatigue, as well as dry cough, and it could be complicated with tiredness, sore throat, and headache. A few of the patients who have COVID-19 do develop symptoms that include stuffy nose, runny nose, as well as diarrhea. The severe form of COVID-19 could progress rapidly into acute respiratory distress syndrome (ARDS).

- The undertaking of reverse transcription-polymerase chain reaction (RT-PCR), as well as next-generation sequencing
(NGS), represents the gold standard for the diagnosis of COVID-19.

- The undertaking of a computed tomography (CT) scan has been for cross-validation. Computed tomography (CT) scan of the thorax has been recommended as the preferred radiology imaging diagnosis option for COVID-19, given its high density and high density as well as high spatial resolution. The common computed tomography (CT) scan of thorax features of include: multiple segmental ground-glass opacities (GGOs), that are distributed predominantly within extra-pulmonary sub-pleural zones as well as along bronchus-vascular bundles with a crazy paving sign as well as inter-lobular thickening of the septum and consolidation. Pleural effusion and mediastinal lymph node enlargement are seen only on rare occasions.

- Computed tomography (CT) scan of thorax imaging features of COVID-19, do manifest differently within the various stages of the disease that include the early stage, the progression/consolidation stage, and the absorption stage. Within its early stage, COVID-19 pneumonia does manifest as scattered flaky GGOs in varying sizes, that is dominated by peripheral pulmonary zone/subpleural distributions. Within the progression stage of COVID-19 pneumonia, GGOs do increase about their number and size, as well as consolidations of the lung could become visible. The main CT scan features of COVID-19 in the absorption stage include interstitial change of both lungs that include fibrous cords, as well as reticular opacities. Differentiation between COVID-19 pneumonia from other viral pneumonia needs to be undertaken, and the undertaking of computed tomography (CT) scan of thorax could help reduce the false-negative results of nucleic acid tests.

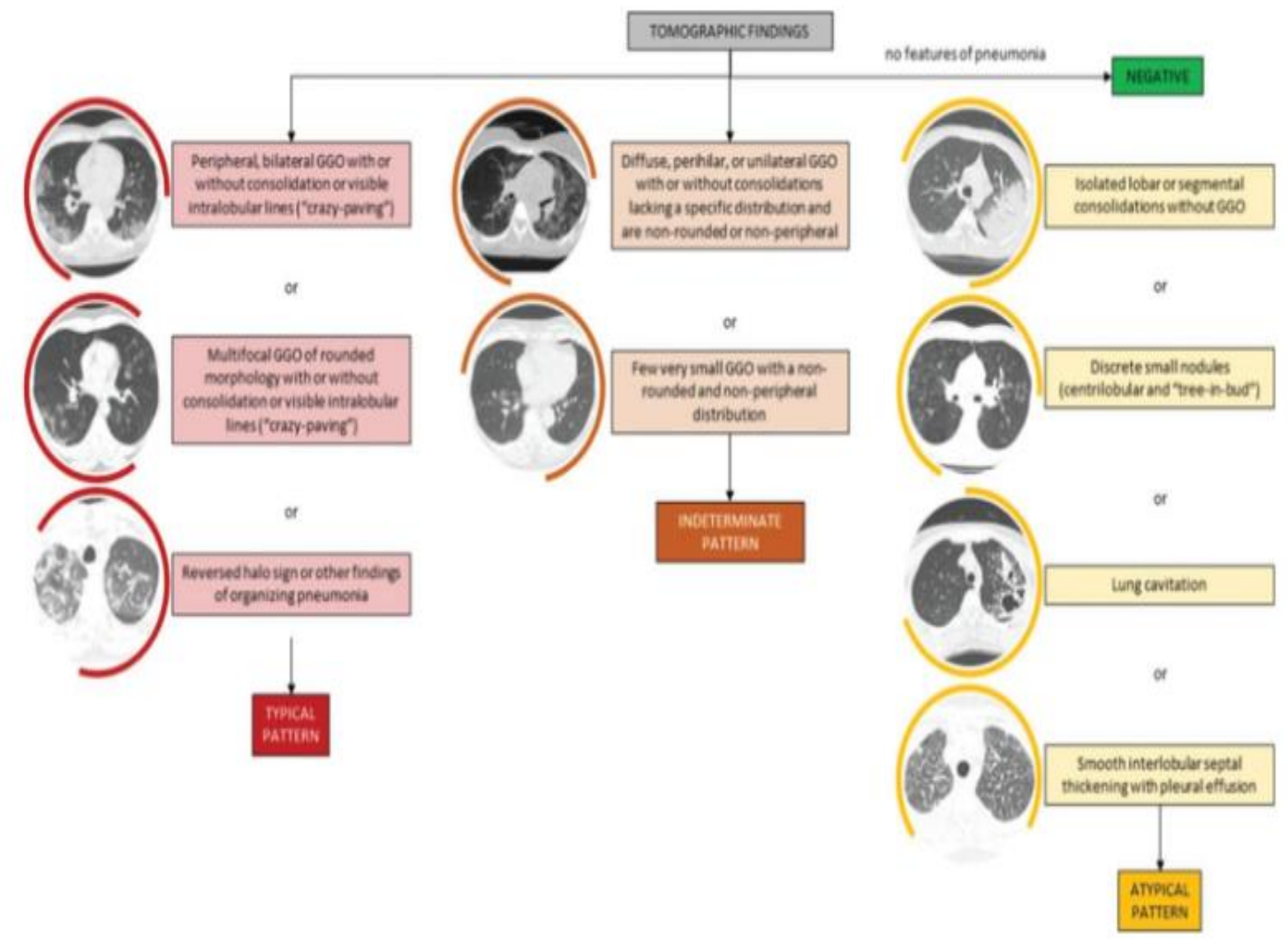

Figure 9: 
Recommendations for categorizing computed tomography findings of COVID-19 pneumonia. Adapted from Simpson et al. [8] [new 30]. Simpson S, Kay FU, Abbara S, Bhalla S, Chung JH, Chung M, et al. Radiological Society of North America Expert Consensus Statement on Reporting Chest CT Findings Related to COVID-19. Endorsed by the
Society of Thoracic Radiology, the American College of Radiology, and RSNA. J Thorac Imaging. 2020. https://doi.org/10.1097/RTI.0000000000000524] and from Da Farias et al. [23]

\section{Table 1 Adapted Proposed Reporting Language for CT Findings Related to COVID-19 used in the Hospital das Clinicas da Faculdade de Medicina da USP.}

\begin{tabular}{|c|c|}
\hline Appearance & Suggested reporting language \\
\hline Typical & $\begin{array}{l}\text { Imaging features are suggestive of pneumonia and viral etiology must be included in the etiological differential diagnosis, particularly } \\
\text { COVID-19. }\end{array}$ \\
\hline Indeterminate & $\begin{array}{l}\text { Imaging features are nonspecific and can be found in a variety of infectious and non-infectious processes, including cases of viral } \\
\text { pneumonia and COVID-19. }\end{array}$ \\
\hline Atypical & $\begin{array}{l}\text { Imaging features suggest pulmonary inflammationlinfection, although its pattern is not usually reported in COVID-19 cases. Other } \\
\text { etiological agents should be considered initially. }\end{array}$ \\
\hline $\begin{array}{l}\text { Negative for } \\
\text { pneumonia }\end{array}$ & No CT findings suggestive of pulmonary inflammation/infection. Note: CT may be negative in some early stages of COVID-19. \\
\hline
\end{tabular}

Table 1.

Table 1. Adapted Proposed Reporting Language for CT Findings Related to COVID-19 used in the Hospital das Clínicas da Faculdade de Medicina da USP Reproduced from Da Farias et al. [23] The typical CT finding for COVID-19 consists of (1) peripheral, bilateral GGO with or without consolidation or visible intralobular lines (crazy-paving pattern); (2) multifocal GGO of rounded morphology with or without consolidation or visible intralobular lines (crazy-paving pattern); or (3) reversed halo sign or other findings of organizing pneumonia. These findings are suggestive of pneumonia, and viral etiology must be included in the etiological differential diagnosis, particularly COVID-19.

Miscellaneous images from our archives that would tend to mimic COVID19 Pneumonia

Pulse Oximeter

A Pulse Oximeter can show lung hypoxia in Covid19 Pneumonia, which appears to be a game-changer in distinguishing it from other causes of Pneumonia and infections.

The following images are a random selection of a series of Chest Radiographs, and computed tomography (CT) scans that show consolidations, lung volume loss, air-bronchograms, Ground-Glass opacification, pleural effusions \& nonspecific linear shadowing. The abnormalities that have been demonstrated below are indistinguishable from COVID 19 Pneumonia on imaging alone. The images are randomly chosen from the author's archives of several hundred $\mathrm{x}$-ray films and computed tomography (CT) scans. A Pulse Oximeter reading for hypoxemia is crucial. The images that have been illustrated below should alert clinicians to the possibility of a diagnosis of COVID19 which they should exclude by undertaking the COVID19 test

\section{Figure 10. Case 1 of our series}

Figure 10 does illustrate a chest x-ray of an individual that had demonstrated collapse/consolidation of the right upper lobe of the lung. This appearance is non-specific, but it tends to be associated with a wide number of differential diagnoses, but COVID19 pneumonia should be the minds of clinicians when they see radiology images as illustrated below (see figure 10). In this particular case, a series of chest X-rays (CXRs) and High-resolution computed tomography scans (HRCTs) showed calcified pleural plaque, and lung parenchymal shadowing in a patient with a history of asbestos exposure, basal linear shadowing, and pericardial effusion (not shown). Nevertheless, it does represent one radiology image feature that should alert clinicians to exclude COVID19 pneumonia. Radiograph reproduced from our archives of Klaus.

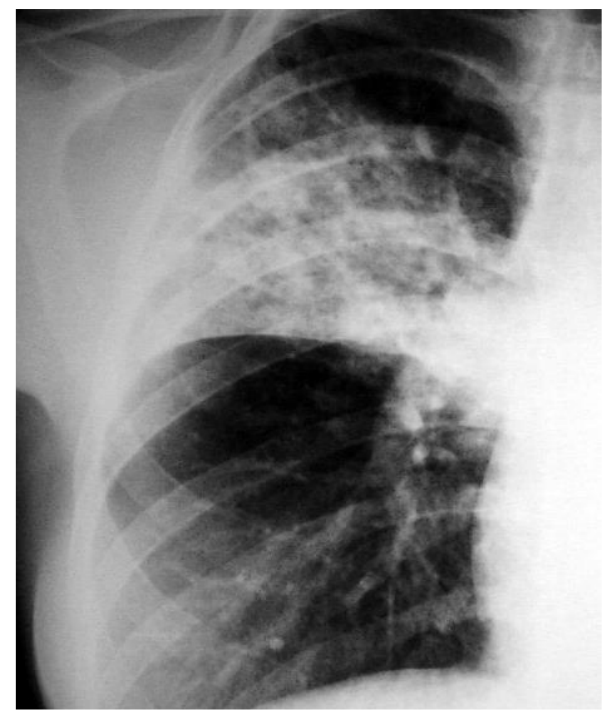

Figure 10: Case 1 of our series.

Collapse/Consolidation right upper lobe; the appearance is nonspecific and has a wide differential. Series of CXRs, \& HRCT showed calcified pleural plaque, and lung parenchymal shadowing in a patient with a history of asbestos exposure, basal linear shadowing, and pericardial effusion (not shown). Radiograph reproduced from our archives of Klaus.

\section{Figure 11: Case 1 from our series}

The illustrated chest $\mathrm{x}$-ray below in figure 4 does illustrate another chest radiograph of the same patient who had a series of chest $\mathrm{x}$-rays and high resolution computed tomography (HRCT) scan of the thorax demonstrated calcified pleural plaque and lung parenchymal shadowing in the patient Case 1 whose initial chest $\mathrm{x}$-ray was shown in figure 10. 


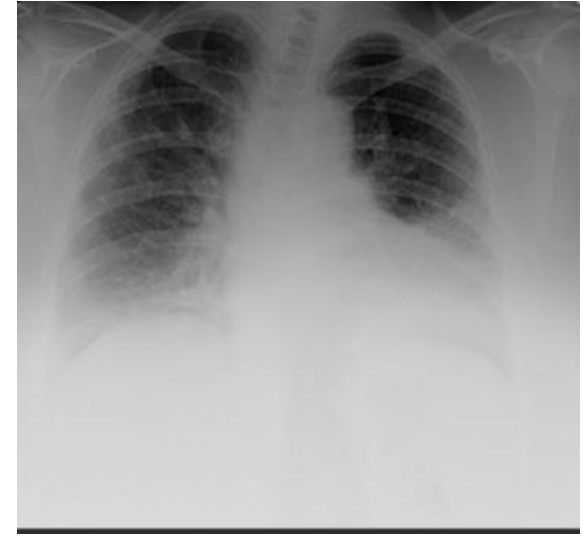

\section{Figure 11}

Figure 11: Case 1 of our series. Series of CXRs, \& HRCT show calcified pleural plaque, and lung parenchymal shadowing in a patient with a history of asbestos exposure, basal linear shadowing, and pericardial effusion. [Radiograph reproduced from our archives of Klaus.

Figure 12: Case 1 of our series.

Figure 12 (see below) has demonstrated a series of High-Resolution Computed Tomography (HRCT) of the thorax which shows calcified pleural plaque, and lung parenchymal shadowing in a patient with a history of asbestos exposure, basal linear shadowing, and pericardial effusion whose chest x-rays were illustrated in figures 9 and 10. Images from personnel archives of Klaus, a co-author of this article.

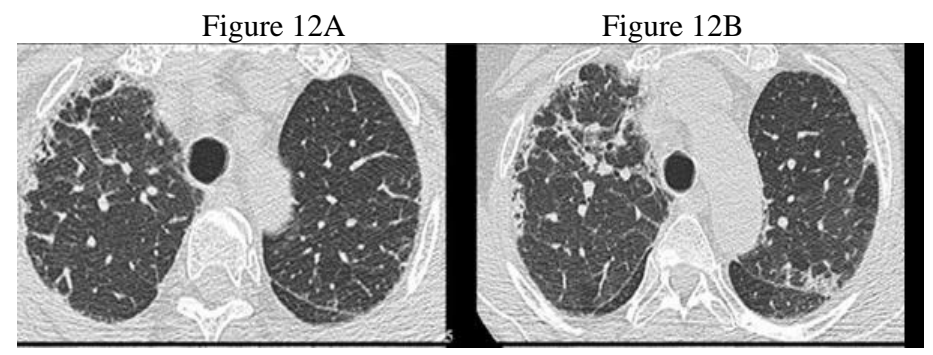

Figure $12 \mathrm{C}$

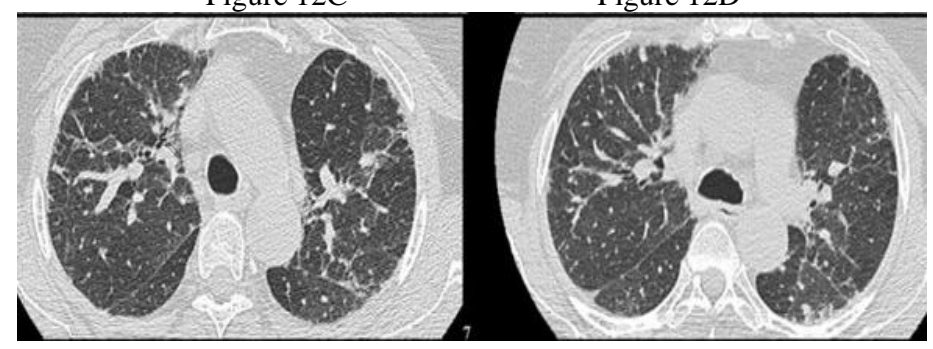

Figure 12: Figures 12A, 12B, 12C, and 12D. HRCT cuts through the mediastinum show calcified pleural plaques, and lung parenchymal shadowing in a patient with a history of asbestos exposure, with basal linear shadowing, and pericardial effusion. Images from personal archives of Klaus, a co-author of this article.

\section{Figure 13: Case 1 from our series.}

Figure 6 (see below) has demonstrated a series of High-Resolution Computed Tomography (HRCT) of the thorax which shows calcified pleural plaque, and lung parenchymal shadowing in a patient with a history of asbestos exposure, basal linear shadowing, and pericardial effusion whose chest $x$-rays were illustrated in figures 3 and 4. Images from personnel archives of Klaus, a co-author of this article.
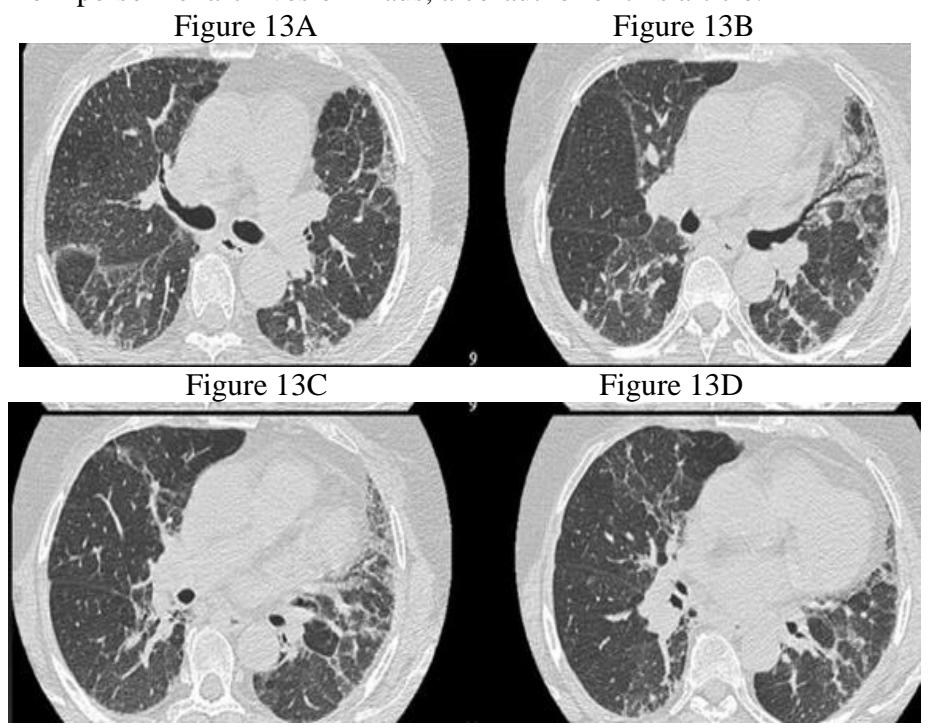

Figures 13. 13A, 13B, 13C, and 123. HRCT cuts through the mediastinum show calcified pleural plaques, and lung parenchymal shadowing in a patient with a history of asbestos exposure, with basal linear shadowing, and pericardial effusion. Images from personal archives of Klaus, a co-author of this article.

Figure 14: Case 1 from our series (see figure 14 below) Figure 14 represents a series of HRCT which shows calcified pleural plaque, and lung parenchymal shadowing in a patient with a history of asbestos exposure, basal linear shadowing, and pericardial effusion. Figure 14 represents High resolution computed tomography (HRCT) images of Case 1, whose chest x-rays and CT scan images have been illustrated in figures $10,11,12$, and 13 . The images have been reproduced from personal archives Klaus et al
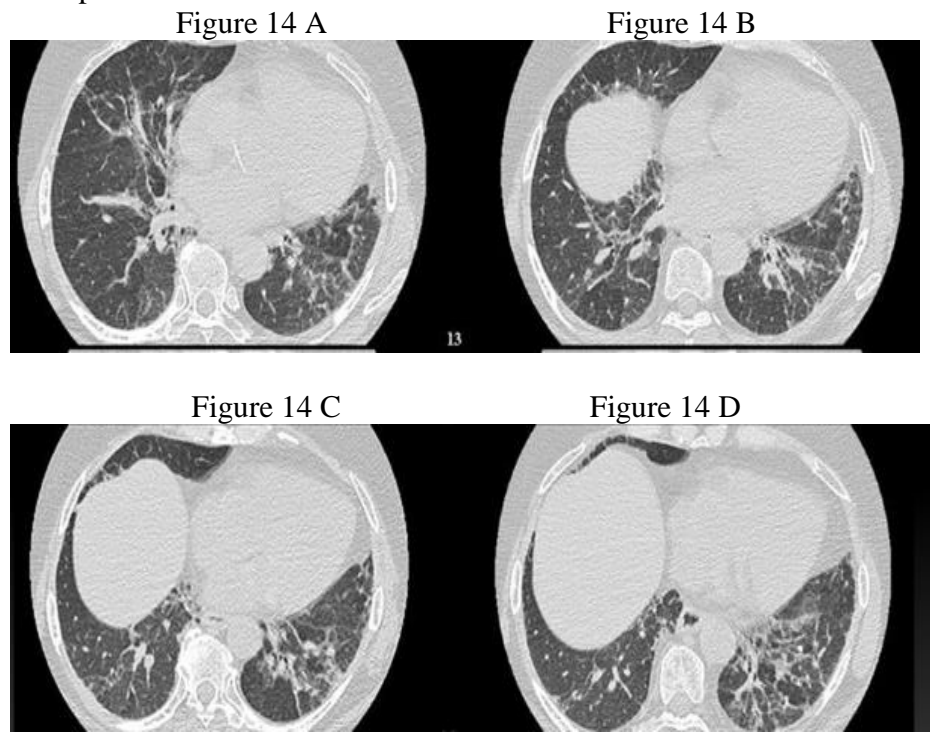

Figure 14: Figures 14A, 14B, 14C, and 14D. HRCT cuts through the mediastinum show calcified pleural plaques, and lung parenchymal shadowing in a patient with a history of asbestos exposure, with basal linear shadowing, and pericardial effusion. Images from personal archives Klaus. 


\section{Figure 15. Case 2}

Figure 15: Case 2 shows a chest x-ray of a patient who had presented with cough, fever, and shortness of breath. The chest Radiograph shows nonspecific bi-basal shadowing, and during the COVID19 pandemic, the finding of non-specific finding as illustrated in figure 15 should alert the clinician to undertake tests to exclude COVI19 pneumonia even though case 2 was not a case of COVID19 but does mimic non-specific feature that may be seen in COVID19 pneumonia. The image was reproduced from the archives of Klaus, a co-author of this article.

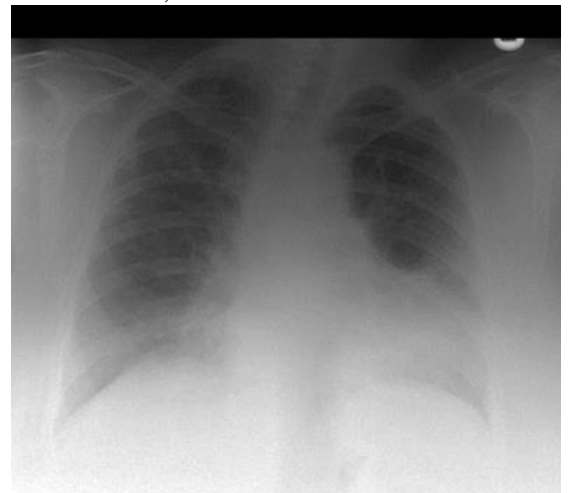

Figure 15. A chest $x$-ray of a patient that presented with cough, fever, and shortness of breath. The chest Radiograph shows nonspecific bi-basal shadowing; the image was reproduced from the archives of Klaus, a coauthor of this article.

\section{Figure 16:}

Figure16 represents axial CT scans on the same patient (case 2) whose chest X-ray (CXR) was illustrated in Figure 15 show extensive calcified pleural plaques (white arrow) but no other lung parenchymal abnormality. The patient's symptoms were un-explained, which settled without intervention. The case was not that of COVID19 but the chest x-ray features are non-specific which could also be seen in some cases of COVID 19 pneumonia and the finding of chest x-ray features as illustrated in figure 15 should alert the clinician to have a high index of suspicion to exclude COVID19 pneumonia by undertaking COVI19 tests. The images have been reproduced from the archives of Klaus, a co-author of this article.

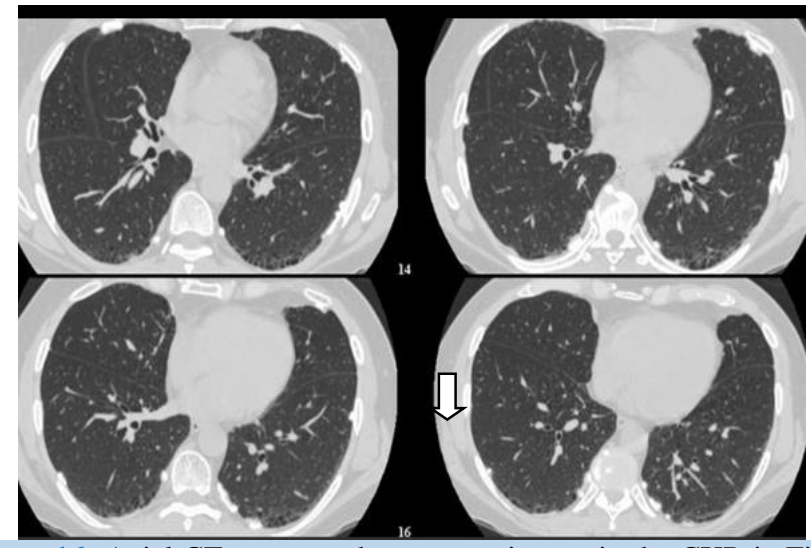

Figure 16: Axial CT scans on the same patient as in the CXR in Figure 15 show extensive calcified pleural plaques (white arrow) but no other lung parenchymal abnormality. The patient's symptoms were unexplained, which settled without intervention-images from personal archives Klaus, a co-author of this article.

\section{Figure 17: Case 3.}

Figure 17 represents a chest x-ray of another patient (case 3) who presented with exertional dyspnea, and the chest $\mathrm{x}$-ray shows ground- glass opacification at the lung bases that had been illustrated by a star. The chest $x$-ray finding, in this case, is non-specific and could be demonstrated in a number of differential diagnoses but the finding of a chest $\mathrm{x}$-ray feature as illustrated in figure 17 should alert the clinician to undertake COVID19 test to confirm or negate the diagnosis of COVID19 pneumonia in the current COVID19 pandemic era.

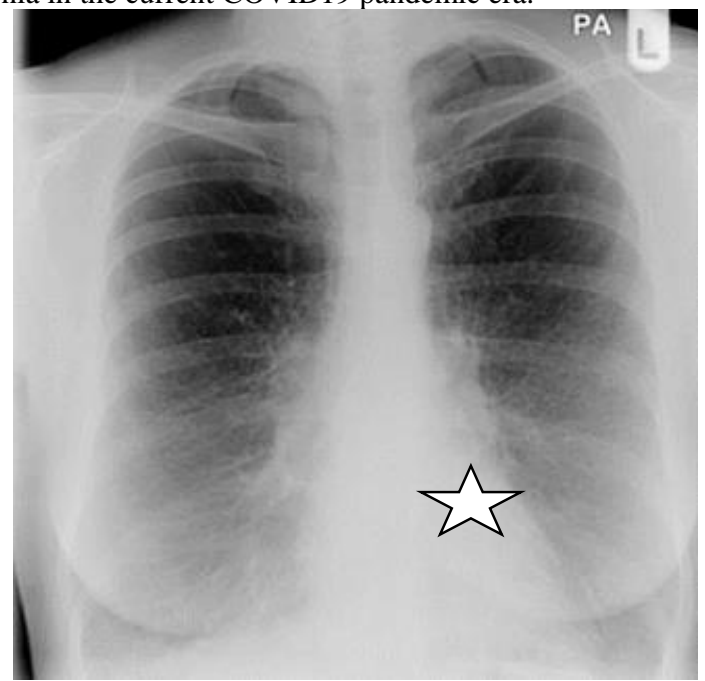

\section{Figure 17}

A radiograph of a patient that presented with exertional dyspnea shows ground-glass opacification at the lung bases (star). The Radiograph was reproduced from the personal archives Klaus, a co-author of this article.

\section{Figure 18: Case 3.}

Figure 18 represents axial CT scans of the same patient (case 3) whose chest x-ray was illustrated in Figure 18, and these show subtle groundglass appearance and sub-pleural reticular shadowing. The cause was Collagen vascular-related lung disease. The features that are demonstrated in the figure are non-specific and should alert the clinician to undertake the COVID19 test to exclude or negate the diagnosis of COVID 19 pneumonia. The figure was reproduced from the archives of Klaus, a co-author of this article.

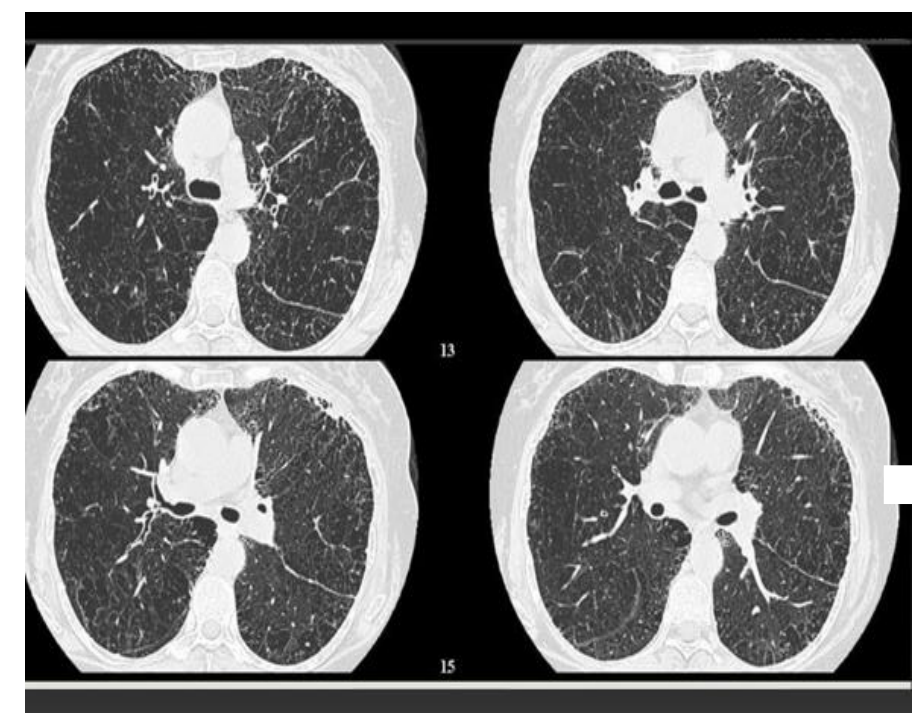

Figure 18. Axial CT scans show subpleural reticular/interstitial shadowing secondary to Collagen vascular-related interstitial lung disease (white arrow). Images reproduced from the archives of Klaus, a co-author of this article. 


\section{Figure 19. Case 3:}

Figure 19 represents axial CT scans of a patient that show subtle ground glass opacification (white arrowhead) secondary to Collagen vascularrelated interstitial lung disease. This CT scan finding is non-specific and could be seen in a variety of pulmonary conditions of which COVID19 is a differential diagnosis, and for this reason, when clinicians see CT scan images that simulate the features that are shown in figure 19, they should undertake COVID19 tests to confirm or negate the diagnosis of COVID19 pneumonia.

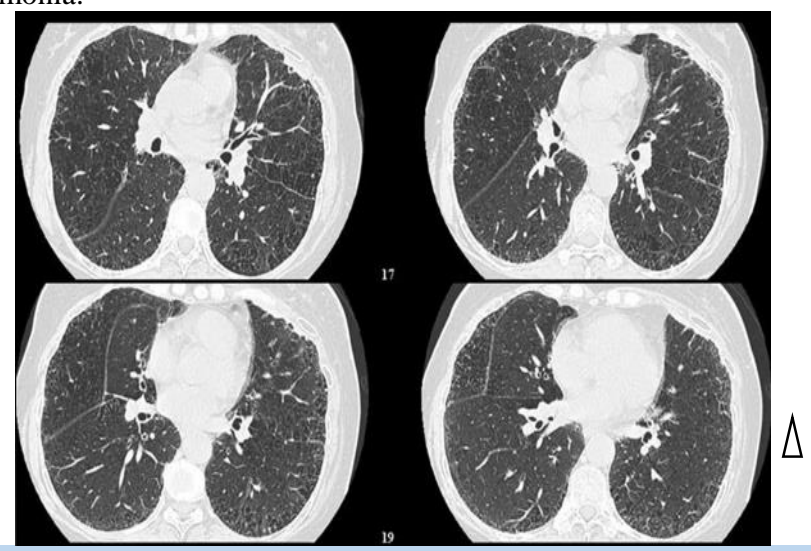

Figure 19. Axial CT scans show subtle ground glass opacification (white arrowhead) secondary to Collagen vascular-related interstitial lung disease. Images Reproduced from the archives of Klaus, a co-author of this article.

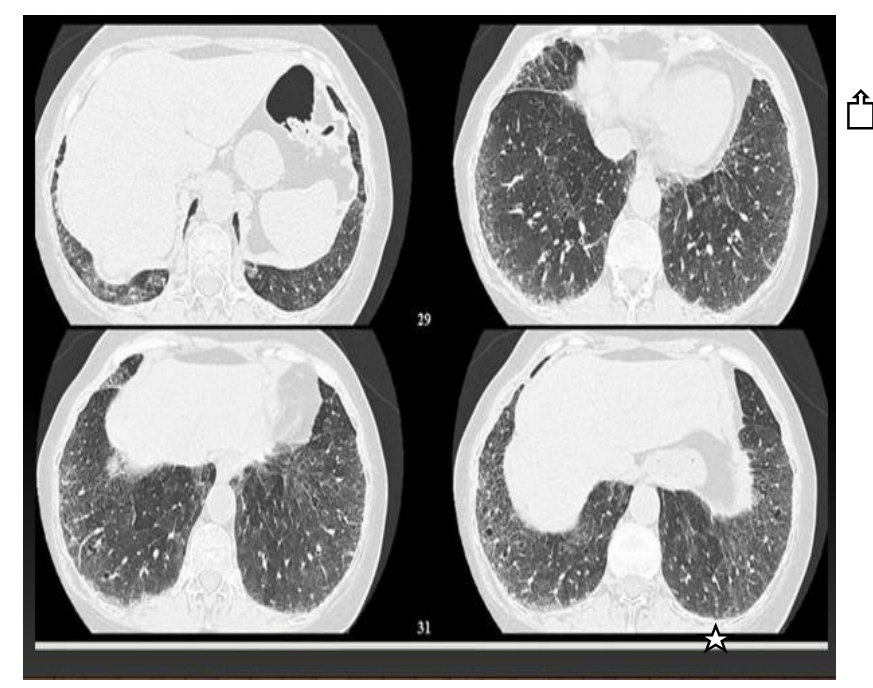

\section{Figure 20}

Figure 20 represents axial CT scans of an individual that shows volume loss within the right upper lobe (truncated white arrow), and reticular shadowing/ground-glass opacification (stars) in both lung fields in a patient who had a biopsy of the lesion and pathology examination of the specimen confirmed the diagnosis of sarcoidosis. The CT scan features that are illustrated in the CT scan are non-specific. The features are shown within the CT scan could be seen in various differential diagnoses of which COVID19 is one of the differential diagnoses. Clinicians need to have a high index of suspicion to exclude COVID19 when they see images that simulate the demonstrated images in figure 13 so that they could undertake COVID19 tests to confirm or negate the diagnosis of COVID19 during the current COVID19 pandemic era. Figure reproduced from the archives of Klaus, a co-author of this article.

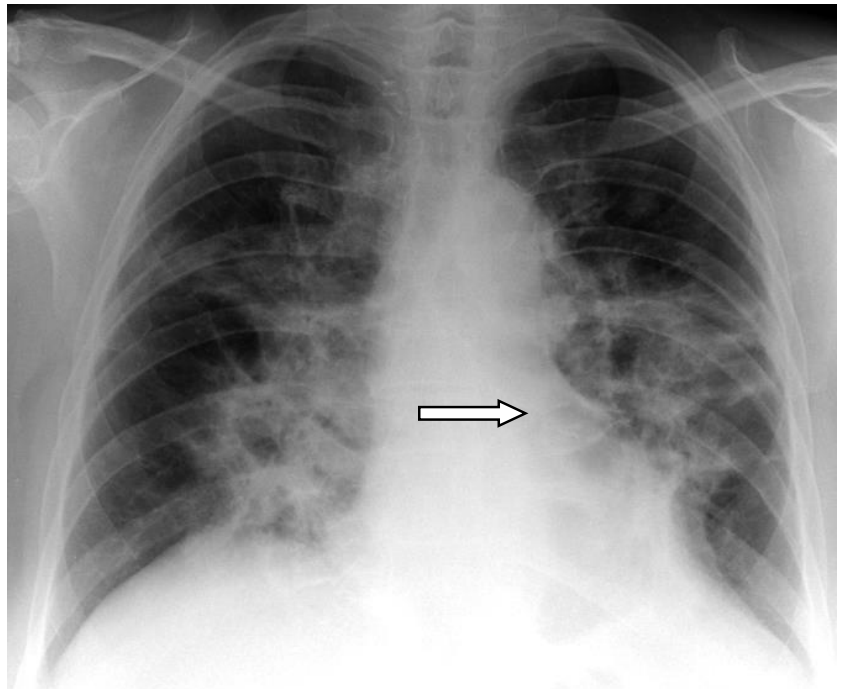

Figure 21

Figure 21 represents a chest $\mathrm{x}$-ray (CXR) of an individual that shows extensive bilateral mid and lower zones shadowing associated with loss of volume (white arrows). The shadowing appears to be fibrotic nature. The individual had a fine needle aspiration biopsy (FNA) of the lesion, and pathology examination of the specimen showed features of old burntout pulmonary sarcoidosis. The chest $\mathrm{x}$-ray features that are illustrated in figure 21 are non-specific because similar features could be demonstrated in a number of differential diagnoses, including COVID19 pneumonia. Therefore clinicians should have a high index of suspicion to exclude COVID19 by undertaking COVID19 tests. Images Reproduced from the archives of Klaus, a co-author of this article.

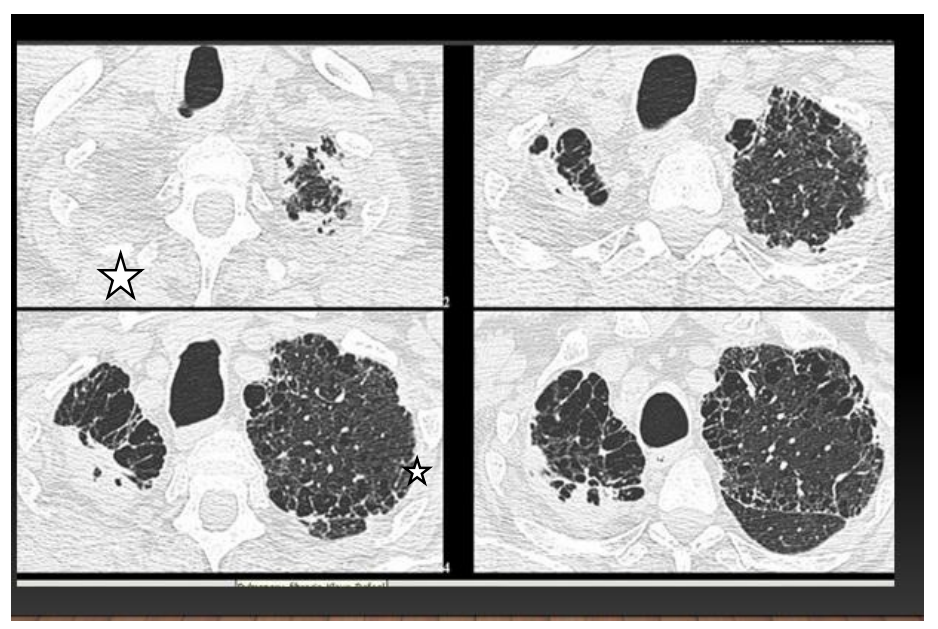

Figure 22

Figure 22 represents axial CT scans of the same patient whose chest x-ray was illustrated in Figure 21 showing extensive fibrosis, amounting to honeycomb appearance, (white obelisk), and marked pleural thickening (white star). The CT scan image features illustrated are non-specific, and features that simulate the features shown in figure 22 could be seen in other conditions, including COVID19. Hence, when CT scans are undertaken that demonstrate features that simulate the features illustrated in figure 22, clinicians would need to have a high index of suspicion to exclude COVID 19 by conducting COVID19 tests-reproduced from the archives of Klaus, a co-author of this article. 

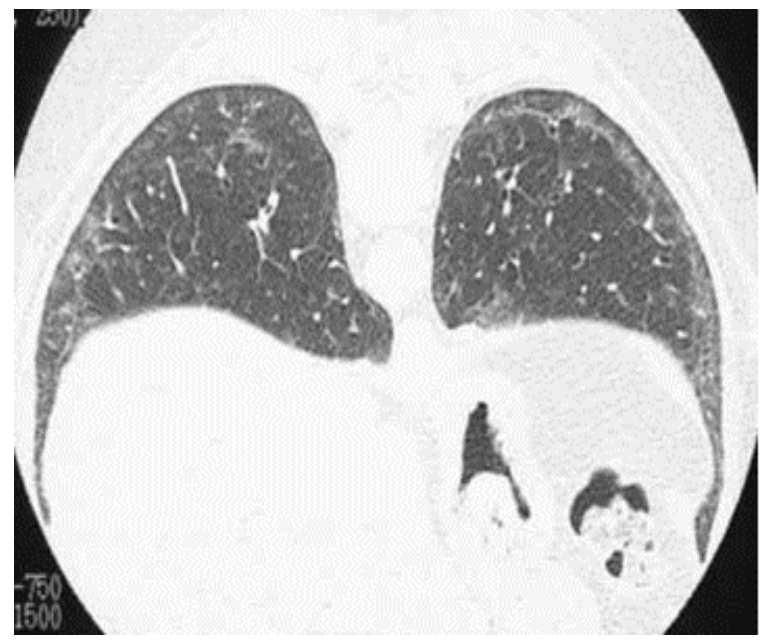

Figure 23

Figure 23, represents a prone Axial CT scan of the thorax of an individual that shows subtle interstitial lung fibrosis due to collagen vascular disease, which shows the value of likely CT scan. The CT scan features that have been demonstrated are non-specific. Features that simulate the described features could be seen in other conditions, and COVID19 is one of the differential diagnoses. For this reason, clinicians that see CT scan features of their patients within the current COVID19 pandemic era should have a high index of suspicion to exclude the diagnosis of COVID19 pneumonia by undertaking COVID19 tests. Figure 23 image was reproduced from the archives of Klaus, a co-author of this article.

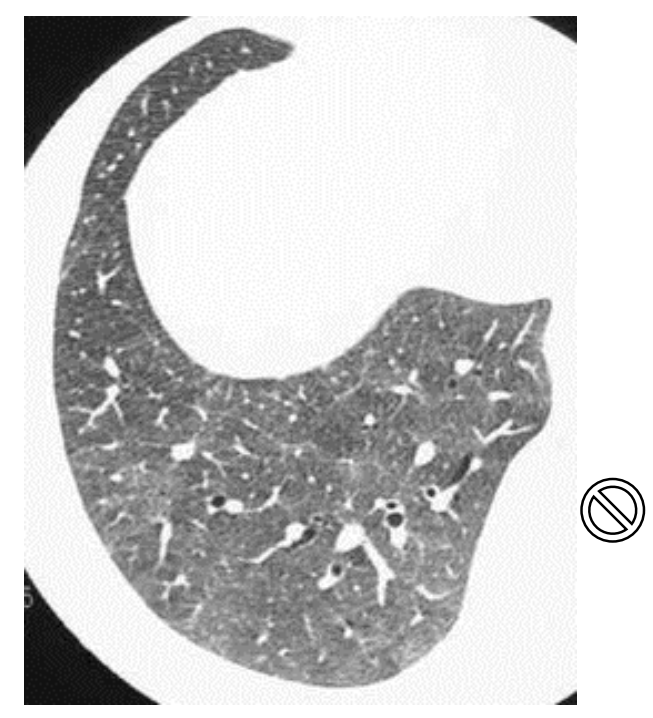

\section{Figure 24}

Figure 24 represents a Non-Enhanced Axial CT scan of the thorax of an individual, which shows extensive ground-glass opacification secondary to hypersensitivity pneumonitis (no entry sign). The features illustrated in the CT scan are non-specific and could be demonstrated in differential diagnosis mimics of the condition including in COVID19 pneumonia and hence within the current COVID19 pandemic era, clinicians whose patients have CT scans that show features as has been illustrated in figure 24 should have a high index of suspicion to exclude the possibility of COVID19 pneumonia by undertaking COVI19 tests. Figure 24 was reproduced from the archives of Klaus, a co-author of this article.

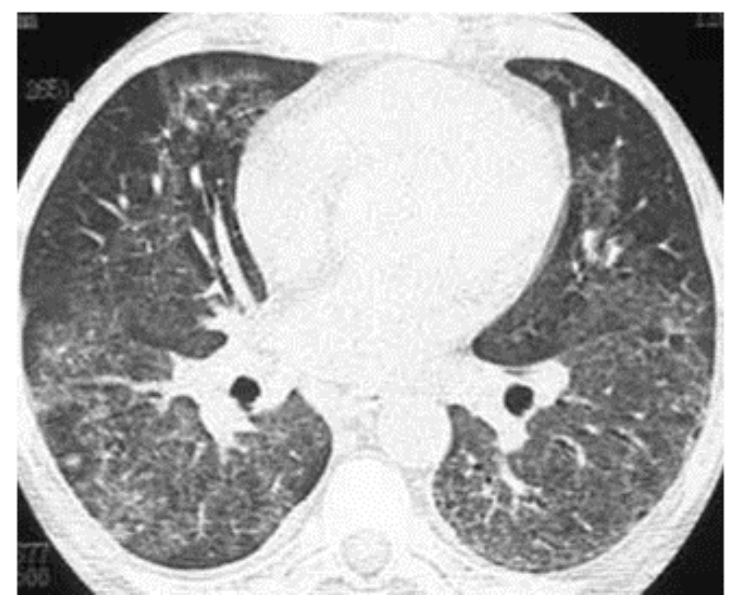

Figure 25

Figure 25 represents an axial CT scan of thorax an individual through the midzone that depicts extensive ground-glass opacification, secondary to hypersensitivity pneumonitis. The features demonstrated in the CT scan are non-specific and could be shown in a number of differential diagnoses, and one of these differential diagnoses is COVID19. Hence when a clinician encounters radiology imaging features that simulate the features that have been illustrated in figure 24 , they should have a high index of suspicion to exclude COVID19 pneumonia by undertaking COVID19 pneumonia. The image was reproduced from the archives of Klaus, a coauthor of this article.

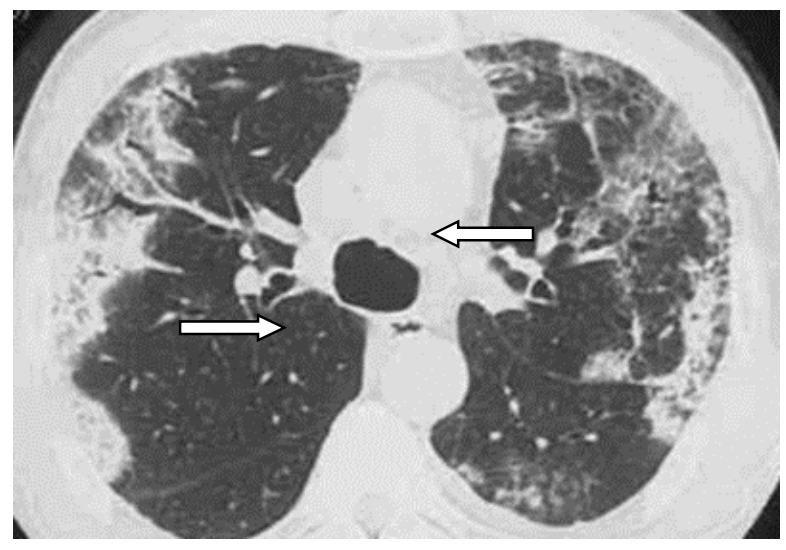

Figure 26

Figure 26 illustrates an unenhanced axial computed tomography (CT) scan through the superior mediastinum of an individual, which shows features of a case of a biopsy-proven chronic eosinophilic Pneumonia. Note the subpleural lung parenchymal shadowing, sparing the paracardial region's image (arrows). The patient's history and laboratory parameters are instrumental in the diagnosis. The features demonstrated in the CT scan of the mediastinum are non-specific because the features could also be shown in various differential diagnoses, and COVID19 is one of such conditions. Clinicians who see CT scan features of their patients that simulate the CT scan features that have been illustrated in figure 26 should have a high index of suspicion for the possibility of COVID19 pneumonitis within the current COVID19 era to undertake COVID19 tests that would confirm or negate the possible diagnosis of COVID pneumonia-reproduced from the archives of Klaus, a co-author of this article. 

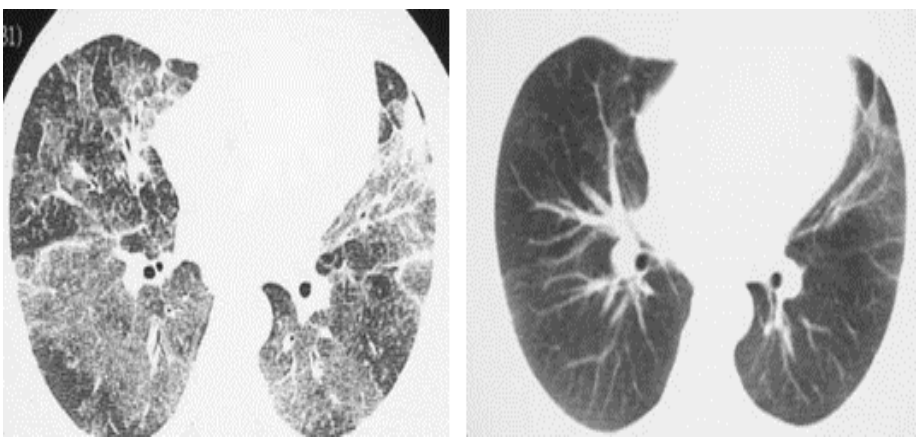

Figure 27

Figure 27 demonstrates the radiology imaging features of an individual who had hypersensitivity pneumonitis due to Amiodarone, axial scans before and after cessation of Amiodarone. The scan on the left shows extensive air-space shadowing in both lung fields mimicking pulmonary edema, which has almost completely cleared after cessation of Amiodarone; only minimal air space shadowing persists in the left paracardial region (white arrow). The image features that have been illustrated in the scans are non-specific. CT scan of thorax features that mimic the features that have been shown in figure 27 can also be seen in a number of differential diagnoses including COVID19 pneumonia and for this reason, clinicians whose patients undergo CT scan of the thorax that demonstrate features that simulate the illustrated features of figure 27 should have a high index of suspicion to exclude the possibility of COVID 19 pneumonia by undertaking COVID19 tests on their patients. The images in Figure 27 have been reproduced from the archives of Klaus, a co-author of this article.
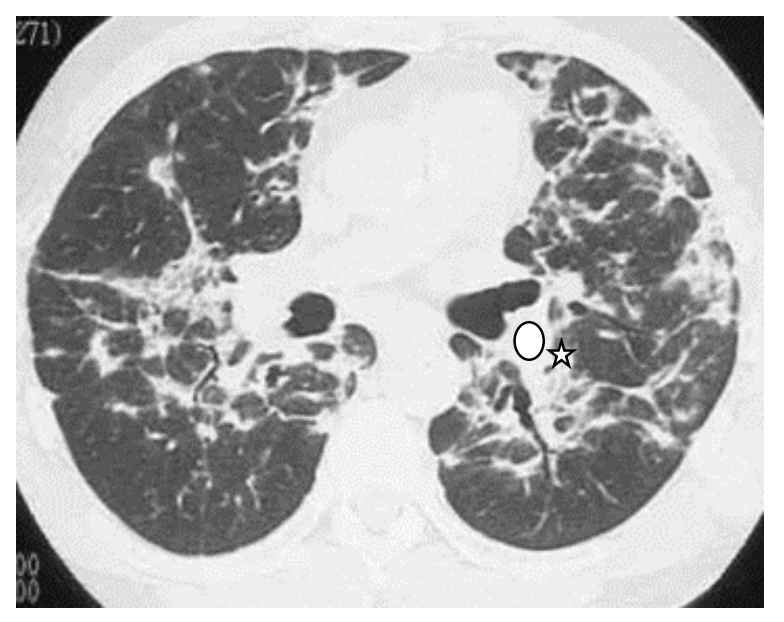

\section{Figure 28}

Figure 28 demonstrated an unenhanced axial computed tomography (CT) scan of the thorax of an individual that has shown linear shadowing of fibrotic nature in a biopsy-proven Cryptogenic Organizing Pneumonia that has been illustrated with white obelisks in mid-zones/lung bases. These features are non-specific and could also be demonstrated in some differential diagnoses, of which COVID19 pneumonia is one of them. Given this within the current COVID19 pandemic era, clinicians should have a high suspicion for the possibility of COVID19 pneumonia when their patients have computed tomography (CT) scans of the thorax that demonstrates features that simulate the features in figure 28 to request the undertaking of COVID19 tests by their patients. The figure was reproduced from the archives of Klaus, a co-author of this article.

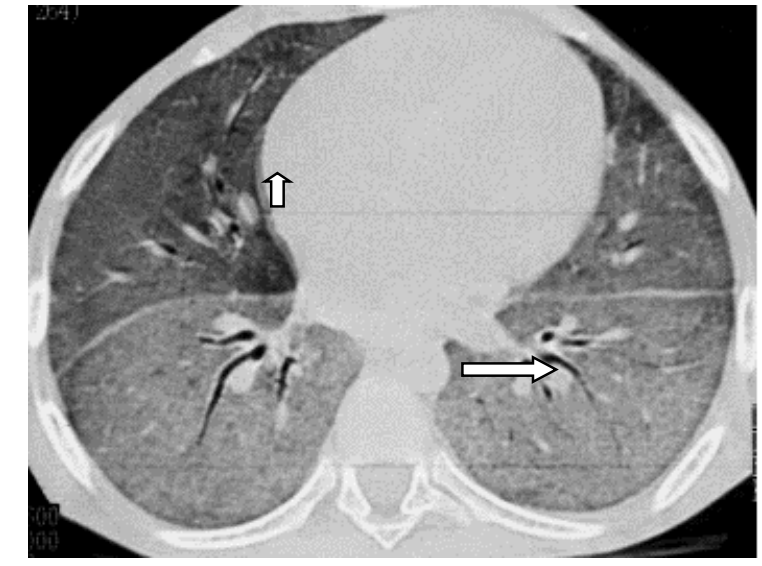

Figure 29

Figure 29 represents axial computed tomography (CT) scans of a patient who had primary Hemosiderosis, note the GGO, and an air-bronchogram at the lung bases (white arrow). This CT scan feature is non-specific and could also be visualized in a number of differential diagnoses, including in COVID19 pneumonia. Given these clinicians whose patients undergo CT scans of the thorax that demonstrate features that mimic the features of figure 29 should have a high index of suspicion to exclude the possibility of the diagnosis of COVID19 in the current COVID19 pandemic era. Figure 29 was reproduced from the archives of Klaus, a coauthor of our article.

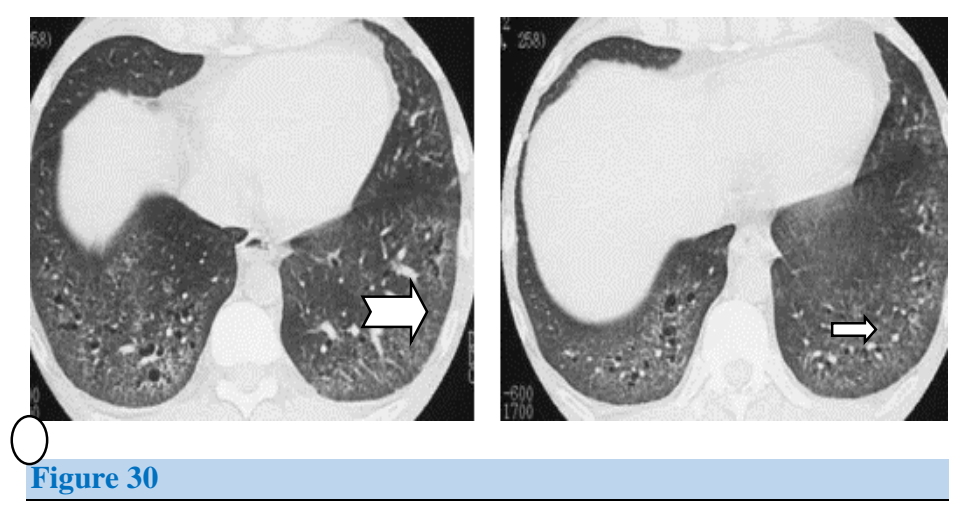

Figure 30. Represents axial non-enhanced computed tomography (CT) scans of the thorax of an individual that shows biopsy-proven mixed connective tissue disease. Note the GGO (white arrow) and submillimeter Lung cysts (thin white arrow). These radiology imaging features are non-specific, and they could be seen in a number of differential diagnoses including COVID19 pneumonia and hence within the current COVID19 pandemic era, clinicians whose patients have CT scans of the thorax that demonstrate features that simulate the features that have been illustrated in figure 30 , should have a high index of suspicion to exclude the possibility of COVID19 pneumonia by requesting that their patients undertake COVID19 tests to confirm or negate the diagnosis of COVID19 pneumonia and to isolate the individuals. Figure 30 was reproduced from the archives of Klaus, a coauthor of our article.

\section{Conclusions}

Many causes of Pneumonia have been described. Pneumonia can occur at any age, and presentation can be non-specific. Various imaging techniques generally diagnose cases of Pneumonia. In the current climate, 
COVID-19 Pneumonia has taken center stage; confirmation relies upon microbiological studies such as real-time polymerase chain reaction or sequencing. These investigations are not usually available in an emergency setting. Computed Tomography (CT) can be used as an essential complement for the diagnosis of COVID-19 Pneumonia in the current epidemic context. But the later may be misleading as other cases of Pneumonia, and interstitial lung disease can easily be confused with COVID-19 Pneumonia. Also, COVID-19 Pneumonia may be missed if not considered.

\section{Conflict of interest: - None}

\section{Acknowledgments}

Acknowledgments to:

- Simpson S, Kay FU, Abbara S, Bhalla S, Chung JH, Chung M, et al. and the Radiological Society of North America Expert Consensus Statement on Reporting Chest CT Findings Related to COVID-19 and the Society of Thoracic Radiology, the American College of Radiology and RSNA J Thoracic Imaging.

- $\quad$ Zhang X, Song W, Liu X, Lyu L. CT and the Japanese Journal of Radiology

- Dai W C, Zhang H W, Yu J, Xu H J, Chen H, Luo SP, Zhang $\mathrm{H}$, Liang L H, Wu X L, Lei Y, Lin F., and the Can Association of Radiology and their Journal.

- Huang P, Liu T, Huang L, Liu H, Lei M, Xu W, Hu X, Chen J, Liu B. and Radiology, and the Radiological Society of North America, Inc.

- $\quad$ Farias L P G, Fonseca EKUN, Strabelli DG, et al. and Clinics (Sao Paulo)

- All medics and paramedical staff who were dealing with lung infections

- Dr. Klaus Irion and Professor Ali Nawaz Khan

\section{References}

1. Hani C, Trieu N H, Saab I, Dangeard S, Bennani S, Chassagnon G, Revell M P. (2020) COVID-19 pneumonia; present A review of typical CT findings and differential diagnosis. Diagn Interv Imaging.

2. European Centre for Disease Prevention and Control -19 Epidemiological pandemic updates and risk assessment.

3. Nickel C H, Bingisser R. (2020) Mimics and chameleons of Covid-19. Swiss Medical Weekly.

4. Shi H, Han X, Jiang N, Cao Y, Alwalid O, Gu J. (2020) Radiological findings from 81 patients with COVID-19 pneumonia in Wuhan, China: a descriptive study. Lancet Infect Dis. S1473-3099(20)30086-30094.

5. Xie X, Zhong Z, Zhao W, Zheng C, Wang F, Liu J. (2020) Chest CT for Typical Coronavirus Disease 2019 (COVID-19) Pneumonia: Relationship to Negative RT-PCR Testing. Radiology. Aug; 296(2): E41-E45.

6. Wang W, Xu Y, Gao R, Lu R, Han K, Wu G. (2020) Detection of SARS-CoV-2 in Different Types of Clinical Specimens. JAMA.

7. Nemec M, Koller MT, Nickel CH, Maile S, Winterhalder C, Karrer C. (2010) Patients are presenting to the emergency department with nonspecific complaints: the Basel Nonspecific Complaints (BANC) study. Acad Emerg Med.17 (3):284-292.

8. Huff J S. (2020) Stroke mimics and chameleons. Emerg Med Clin North Am.20 (3):583-595.

9. Salathé M, Althaus C L, Neher R, Stringhini S, Hodcroft E, Fellay J, Zwahlen M, Senti G, Battegay M. (2020) COVID-19 epidemic in Switzerland: on the importance of testing, contact tracing, and isolation. Swiss Med Wkly.

10. Battegay M, Kuehl R, Tschudin-Sutter S, Hirsch HH, Widmer AF, Neher RA. (2020) 2019-novel Coronavirus (2019-nCoV): estimating the case fatality rate - a word of caution. Swiss Med Wkly.

11. Li Y H, Shen L, Li J. (2020) Chemotherapy strategy for colorectal cancer under the outbreak of coronavirus disease 2019; Zhonghua Wei Chang Wai Ke Za Zhi Mar 25: 23(3): 217-219.

12. Chen L, Liu HG, Liu W, Liu J, Liu K, Shang J, Deng Y, Wei S. (2020) Analysis of clinical features of 29 patients with 2019 novel coronavirus pneumonia. Zhonghua Jie He He Hu Xi Za Zhi. Mar 12; 43(3):203-208.

13. A Peng A, Song P, Lian K, Wang Y. (2020) CT Manifestations of Novel Coronavirus Pneumonia: A Case Report. Balkan Med J. Apr 37(3):163-165.

14. Zhang X, Song W, Liu X, Lyu L. (2020) CT image of novel coronavirus pneumonia: a case report. Jpn J Radiol. Mar 18: 12.

15. Huang P, Liu T, Huang L, Liu H, Lei M, Xu W, Hu X, Chen J, Liu B. (2020) Use of Chest CT in Combination with Negative RT-PCR Assay for the 2019 Novel Coronavirus but High Clinical Suspicion. Radiology. 295(1):22-23.

16. Pan Y, Guan H, Zhou S, Wang Y, Li Q, Zhu T, Hu Q, Xia L. (2020) Initial CT findings and temporal changes in patients with the novel coronavirus pneumonia (2019-nCoV): a study of 63 patients in Wuhan, China. J Eur Radiol.

17. Zhu N, Zhang D, Wang W, Li X, Yang B, Song J, Zhao X, Huang B, Shi W, Lu R, Niu P, Zhan F, Ma X, Wang D, Xu W, Wu G, Gao G F, Tan W. (2020) China Novel Coronavirus Investigating and Research Team. A Novel Coronavirus from Patients with Pneumonia in China, 2019. N Engl J Med. Feb; 382(8):727-733.

18. FDA. (2020) Fact sheet for healthcare providers: CDC - 2019nCoV Real-Time RT-PCR Diagnostic Panel.

19. Song F, Shi N, Shan F, Zhang Z, Shen J, Lu H, Ling Y, Jiang Y, Shi Y. (2020) Emerging 2019 Novel Coronavirus (2019nCoV) Pneumonia. Radiology. Apr; 295(1):210-217.

20. Dai W C, Zhang H W, Yu J, Xu H J, Chen H, Luo SP, Zhang H, Liang L H, Wu X L, Lei Y, Lin F. (2020) CT Imaging and Differential Diagnosis of COVID-19. Can Assoc Radiol. May; 71(2): 195-200.

21. Xu X, Yu C, Qu J, Zhang L, Jiang S, Huang D, Chen B, Zhang Z, Guan W, Ling Z, Jiang R, Hu T, Ding Y, Lin L, Gan Q, Luo L, Tang X, Liu J. (2020) Imaging and clinical features of patients with 2019 novel coronavirus SARS-CoV-2 Eur J Nucl Med Mol Imaging. May; 47(5):1275-1280.

22. Farias L PG, Fonseca EKUN, Strabelli DG, et al. (2020) Imaging findings in COVID-19 pneumonia. Clinics (Sao Paulo).

23. ACR Recommendations for the use of Chest Radiography and Computed Tomography (CT) for Suspected COVID-19 Infection.

24. Choi H, Qi X, Yoon SH, Park SJ, Lee KH, Kim JY, et al. (2020) Extension of Coronavirus Disease 2019 (COVID-19) on Chest CT and Implications for Chest Radiograph Interpretation. Radiology: Cardiothoracic Imaging.2 (2).

25. Rubin GD, Ryerson CJ, Haramati LB, Sverzellati N, Kanne JP, Raoof S, et al. (2020) The Role of Chest Imaging in Patient Management During the COVID-19 Pandemic: A Multinational Consensus Statement from the Fleischner Society. Chest. 
26. Wong HYF, Lam HYS, Fong AH, Leung ST, Chin TW, Lo CSY, et al. (2020) Frequency and Distribution of Chest Radiographic Findings in COVID-19 Positive Patients. Radiology.

27. Recomendações de uso de métodos de imagem para pacientes suspeitos de infecção pelo COVID-19.

28. Grupo força colaborativa COVID-19 Brasil. Orientações sobre Diagnóstico, Tratamento e Isolamento de Pacientes com COVID-19.

29. Simpson S, Kay FU, Abbara S, Bhalla S, Chung JH, Chung M, et al. (2020) Radiological Society of North America Expert Consensus Statement on Reporting Chest CT Findings Related to COVID-19. Endorsed by the Society of Thoracic Radiology, the American College of Radiology, and RSNA. J Thorac Imaging.

30. Society of Thoracic Radiology. STR/ASER COVID-19 Position Statement, March 11, 2020.

31. Ai T, Yang Z, Hou H, Zhan C, Chen C, Lv W, et al. (2020) Correlation of Chest CT and RT-PCR Testing for Coronavirus Disease 2019 (COVID-19) in China: A Report of 1014 Cases. Radiology.

32. Bernheim A, Mei X, Huang M, Yang Y, Fayad ZA, Zhang N, et al. (2020) Chest CT Findings in Coronavirus Disease-19 (COVID-19): Relationship to Duration of Infection. Radiology.295 (3).

33. Pan F, Ye T, Sun P, Gui S, Liang B, Li L, et al. (2020) Time Course of Lung Changes at Chest CT during Recovery from Coronavirus Disease 2019 (COVID-19). Radiology. 295(3):715-721.
34. Salehi S, Abedi A, Balakrishnan S, Gholamrezanezhad A. (2020) Coronavirus Disease 2019 (COVID-19): A Systematic Review of Imaging Findings in 919 Patients. AJR Am J Roentgenol.

35. Farias L P G, Strabelli D G, Fonseca E K U N, Loureiro B M C, Nomura C H, Sawamura M V Y. (2020) Alterações tomográficas torácicas em pacientes sintomáticos respiratórios com a COVID-19. Radiol Bras.

36. Farias L PG, Strabelli D G, Sawamura M V Y. (2020) COVID19 pneumonia and the reversed halo sign. J Bras Pneumol.46 (2).

37. Farias L PG, Pereira H A-C, Anastacio E P Z, Minenelli F F, Teles G B D S. (2020) The halo sign as a chest computed tomography finding of COVID-19. Einstein.18: eAI5742.

38. Araujo-Filho J A B, Sawamura M V Y, Costa A N, Cerri G G, Nomura C H. (2020) COVID-19 pneumonia: what is the role of imaging in diagnosis? J. Bras Pneumol. 46(2).

39. Prokop M, van Everdingen W, van Rees Vellinga T, Quarles van Ufford J, Stöger L, Beenen L, et al. (2020) CO-RADS - A categorical CT assessment scheme for patients with suspected COVID-19: definition and evaluation. Radiology.

40. Shoji H, Fonseca E K U N, Teles G B D S, Passos RBD, Yanata E, Silva M M A, et al. (2020) Structured thoracic computed tomography report for COVID-19 pandemic. Einstein.

41. Zheng Q, Lu Y, Lure F, Jaeger S, Lu P. (2020) Clinical and radiological features of novel coronavirus pneumonia. J Xray Sci Technol.28 (3):391-404.

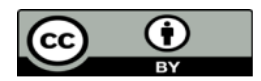

This work is licensed under Creative Commons Attribution 4.0 License

To Submit Your Article Click Here: Submit Article

DOI: $10.31579 / 2692-9406 / 019$
Ready to submit your research? Choose Auctores and benefit from:

$$
\begin{aligned}
& \text { *ast, convenient online submission } \\
& \text { rigorous peer review by experienced research in your field } \\
& \text { rapid publication on acceptance } \\
& \text { * authors retain copyrights } \\
& \text { * imique DOI for all articles } \\
& \text { immediate, unrestricted online access }
\end{aligned}
$$

At Auctores, research is always in progress.

Learn more www.auctoresonline.org/journals/biomedical-researchand-clinical-reviews-- 